\title{
Steady state particle swarm
}

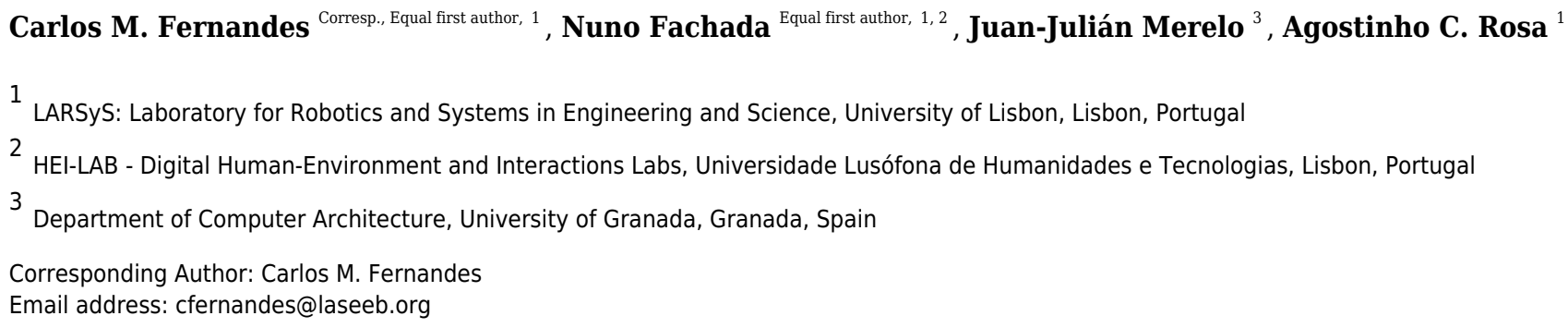

This paper investigates the performance and scalability of a new update strategy for the Particle Swarm Optimization (PSO) algorithm. The strategy is inspired by the Bak-Sneppen model of co-evolution between interacting species, which is basically a network of fitness values (representing species) that change over time according to a simple rule: the least fit species and its neighbors are iteratively replaced with random values. Following these guidelines, a steady state and dynamic update strategy for PSO algorithms is proposed: only the least fit particle and its neighbors are updated and evaluated in each time-step; the remaining particles maintain the same position and fitness, unless they meet the update criterion. The steady state PSO (SS-PSO) was tested on a set of unimodal, multimodal, noisy and rotated benchmark functions, significantly improving the quality of results and convergence speed of the standard PSOs and more sophisticated PSOs with dynamic parameters and neighborhood. A sensitivity analysis of the parameters confirms the performance enhancement with different parameter settings and scalability tests show that the algorithm behavior is consistent throughout a substantial range of solution vector dimensions. 


\section{Steady State Particle Swarm}

2

3 Carlos M. Fernandes ${ }^{1}$, Nuno Fachada ${ }^{2,1}$, Juan-Julián Merelo ${ }^{3}$, Agostinho C. Rosa ${ }^{1}$

4

$5 \quad{ }^{1}$ LARSyS: Laboratory for Robotics and Systems in Engineering and Science, University of

6 Lisbon, Lisbon, Portugal

$7 \quad{ }^{2}$ HEI-LAB - Digital Human-Environment and Interactions Labs, Universidade Lusófona de

8 Humanidades e Tecnologias, Lisbon, Portugal

$9{ }^{3}$ Department of Computer Architecture, University of Granada, Granada, Spain

10

11

12

13

14

15

16

17

18

19

20

21

22

23

24

25

26

27

28

29

30

31

32

33

34

35

36

37

38

39

40

41

Corresponding Author:

Carlos M. Fernandes ${ }^{1}$

Av. Rovisco Pais, 1, Torre Norte, 6.21, Lisboa, Portugal

Email address: cfernandes@laseeb.org

\section{Abstract}

This paper investigates the performance and scalability of a new update strategy for the Particle Swarm Optimization (PSO) algorithm. The strategy is inspired by the Bak-Sneppen model of co-evolution between interacting species, which is basically a network of fitness values (representing species) that change over time according to a simple rule: the least fit species and its neighbors are iteratively replaced with random values. Following these guidelines, a steady state and dynamic update strategy for PSO algorithms is proposed: only the least fit particle and its neighbors are updated and evaluated in each time-step; the remaining particles maintain the same position and fitness, unless they meet the update criterion. The steady state PSO (SS-PSO) was tested on a set of unimodal, multimodal, noisy and rotated benchmark functions, significantly improving the quality of results and convergence speed of the standard PSOs and more sophisticated PSOs with dynamic parameters and neighborhood. A sensitivity analysis of the parameters confirms the performance enhancement with different parameter settings and scalability tests show that the algorithm behavior is consistent throughout a substantial range of solution vector dimensions.

\section{Introduction}

Particle Swarm Optimization (PSO) is a social intelligence model for optimization and learning (Kennedy \& Eberhart, 1995) that uses a set of position vectors (or particles) to represent candidate solutions to a specific problem. Every particle is evaluated by computing its fitness, after its speed and position are updated according to local and global information about the search. During the search, the particles move through the fitness landscape of the problem, following a simple set of equations that define the velocity (equation 1) and position (equation 2) of each particle in each time step and drive them heuristically towards optimal regions of a $D$ dimensional search space. Here, equations 1 and 2 describe a variant proposed by Shi and Eberhart (1999) that is widely used in PSO implementations. The difference to the original PSO 
42 is the introduction of the inertia weight parameter $\omega$ in order to help (together with $c_{1}$ and $c_{2}$ )

43 fine-tuning the balance between local and global search. All PSO implementations in this paper 44 use inertia weight. The velocity $v_{i, d}$ and position $x_{i, d}$ of the $d$-th dimension of the $i$-th particle are 45 therefore updated as follows:

46

$$
\begin{gathered}
v_{i, d}(t)=\omega v_{i, d}(t-1)+c_{1} r 1_{i, d}\left(\text { pbest }_{i, d}-x_{i, d}(t-1)\right)+c_{2} r 2_{i, d}\left(\text { gbest }_{i, d}-x_{i, d}(t-1)\right) \\
x_{i, d}(t)=x_{i, d}(t-1)+v_{i, d}(t)
\end{gathered}
$$

47

48

49

50

51

52

53

54

55

56

57

58

59

60

61

62

63

64

65

66

67

68

69

70

71

72

73

74

75 where $\vec{X}_{i}=\left(x_{i, 1}, x_{i, 2}, \ldots x_{1, D}\right)$ is the position vector of particle $i ; \vec{V}_{i}=\left(v_{i, 1}, v_{i, 2}, \ldots v_{1, D}\right)$ is the velocity of particle $i ; \vec{p}_{\text {besti }}=\left(p_{\text {besti,1 }}, p_{\text {besti,2 } 2}, \ldots p_{\text {best } 1, D}\right)$ is the best solution found so far by particle $i ; \vec{g}_{\text {besti }}=\left(g_{\text {besti,1 }}, g_{\text {besti,2 }}, \ldots g_{\text {best } 1, D}\right)$ is the best solution found so far by the neighborhood of particle $i$. The neighborhood of a particle is defined by the network configuration that connects the population and structures the information flow. Parameters $r 1_{i, d}$ and $r 2_{i, d}$ are random numbers uniformly distributed within the range $[0,1]$ and $c_{1}$ and $c_{2}$ are the acceleration coefficients, which are used to tune the relative influence of each term of the formula.

Most of the PSOs use one of two simple sociometric principles for constructing the neighborhood network (which defines the $\vec{g}_{\text {besti }}$ values). Gbest (where $g$ stands for global) connects all the members of the swarm to one another. The degree of connectivity of gbest is $k=n$, where $n$ is the number of particles. Lbest (where $l$ stands for local), creates a neighborhood with the particle itself and its $k$ nearest neighbors. A particular case of the lbest topology is the ring structure, in which the particles are arranged in a ring, with a degree of connectivity $k=3$, including the particle itself. Between the $k=3$ connectivity of lbest ring and $k=n$ of gbest, there are several possibilities. Two of the most used are the 2-dimensional square lattices with von Neumann and Moore neighborhoods.

Usually, PSOs are synchronous, meaning that first, the fitness values of all vectors must be computed, and only then their velocity is updated. However, there is another possible approach, in which the velocity of the particles is updated immediately after computing the fitness. In this case, the particles move with incomplete knowledge about the global search: if, for instance, the underlying network connecting the particles is a regular graph, then, on average, each particle is updated knowing the current best position found by half of its neighbors and the previous best found by the other half. This variant, which is called asynchronous PSO (A-PSO), was tested by Carlisle and Dozier (2001). In the paper, the authors claim that A-PSO yields better results than the synchronous version (i.e., S-PSO), but since then other authors reached different conclusions: Engelbrecht (2013) and Rada-Vilela et al. (2013), for instance, reported that S-PSO is better than A-PSO in terms of the quality of the solutions and convergence speed. 
The importance of investigating update strategies for PSO lies in the possibility of distributed computation (McNabb, 2014). Even though standard PSOs can be easily parallelized - a particle or a set of particles can be assigned to each processor, for instance -, load imbalances may cause an inefficient use of the computational resources if synchronous updates are used. Asynchronous strategies do not require that all particles in the population have perfect knowledge about the search before the update step (a requirement that may cause idle processor times in a synchronous implementation), and therefore are a valid approach for parallelizing particle swarms. In addition, asynchronism can also be useful in preventing premature convergence (Aziz et all. 2014), or to speed up convergence by skipping function evaluations (Majercik, 2013).

Here, we are mainly concerned with performance issues, in general, and convergence speed in particular. The goal is to design an asynchronous PSO that, unlike the standard A-PSO, significantly improves on the convergence speed of S-PSO in a wide range of problems. We hypothesize that reducing the number of evaluations in each time step, while focusing only on harder cases (i.e, worst solutions), reduces the total number of evaluations required to converge to a specific criterion, i.e., the computational effort to reach a solution. With that objective in mind, we have designed and implemented a novel strategy for one of the fundamental mechanisms of PSO: the velocity update strategy. Following the nature of the method, the algorithm has been entitled steady state PSO (SS-PSO).

In systems theory, a system is said to be in steady state when some of its parts do not change for a period of time (Baillieul \& Samad, 2015). SS-PSO only updates and evaluates a fraction of the population in each time step: the worst particles and its neighbors, thus imposing a kind of selection pressure upon the whole population. The other particles remain in the same position until they eventually fulfill the criterion (being the worst particle or one of its neighbors).

Steady state replacement strategies are common in other population-based metaheuristics, namely Evolutionary Algorithms (Whitley \& Kauth, 1988). However, steady state populations are much less frequent in PSO (Majercik, 2013; Fernandes et al., 2014; Allmendiger et al, 2008). In fact, the strategy proposed in this paper is, to the extent of the authors' knowledge, the first that uses dynamic steady state update coupled with selective pressure. Furthermore, results demonstrate that the criterion for selecting the pool of individuals to update is very important for the success of the update strategy: the update step should be restricted to the worst individuals and their neighbors for optimizing performance. With this design, the steady state update strategy is not only able to improve the convergence speed of PSO standard configurations, but also more sophisticated variants of the algorithm, such as PSOs with time-varying parameters (Ratnaweera et al., 2004) and dynamic neighborhood (Vora \& Mirnalinee, 2017).

The strategy was inspired by the Bak-Sneppen model of co-evolution between interacting species and by the theory of Self-Organized Criticality (SOC) (Bak \& Sneppen, 1993). SOC is a property of some systems that have a critical point as an attractor. However, unlike classical phase transitions, where a parameter needs to be tuned for the system to reach critical point, SOC systems spontaneously reach that critical state between order and randomness. In a SOC system 
115 near the critical point, small disturbances can cause changes of all magnitudes. These events,

116

117

118

119

120

121

122

123

124

125

126

127

128

129

130

131

132

133

134

135

136

137

138

139

140

141

142

143

144

145

146

147

148

149

150

151

152

153

which are spatially or temporally spread through the system, are known as avalanches.

Avalanches occur independently of the initial state. Moreover, the same perturbation may cause small or large avalanches, depending on the current state of the system - i.e., its proximity to the critical point. The distribution of avalanches during a large period displays a power-law between their size and frequency: small avalanches occur very often while large events that reconfigure almost the entire system are scarcer. SOC complex systems balance between stability and creative destruction. In fact, power-law relationships between the size of events and their frequency, one of SOC's signatures, are widespread in Nature. Earthquake distribution, for instance, follows the Gutenberg-Richter law (Gutenberg \& Richter, 1956), a power-law proportion between the magnitude of the earthquakes that occurred in a specific area during a specific period of time, and the frequency of those earthquakes.

SOC was studied for the first time in the sandpile model (Bak et al., 1987). Since then, the concept has been extended to other complex systems: besides the aforementioned earthquakes, the proponents of the theory claim that SOC may be a link between a broad range of phenomena, like forest-fires, ecosystems, financial markets and the brain (Bak, 1996). One of such systems is the Bak-Sneppen model of co-evolution between interacting species (Bak \& Sneppen, 1993).

The Bak-Sneppen model was developed with the main objective of trying to understand the mechanisms underlying mass extinctions in nature. Ecosystems are complex adaptive systems in which the agents (the natural species) are related through several features, like food chains or symbiosis, for instance. In such interconnected environments, the extinction of one species affects the species that are related to it, in a chain reaction that can be of any size: in fact, fossil records suggest that the size of extinction outbreaks is in power-law proportion to their frequency.

In order to model the extinction patterns in nature and search for SOC signatures in coevolutionary systems, Bak \& Sneppen (1993) structured a set of species in a ring network and assigned a fitness value to each. Then, in every time step, the least fit species and its neighbors are eliminated from the system and replaced by individuals with random fitness. To put it in mathematical terms, the system is defined by $n^{d}$ fitness values $f_{i}$ arranged on a $d$-dimensional lattice (ecosystem) with $n$ cells. At each time step, the smallest $f$ value and its $2 \times d$ neighbours are replaced by uncorrelated random values drawn from a uniform distribution. Operating with this set of rules, the system is driven to a critical state where most species have reached a fitness value above a certain threshold. Near the critical point, extinction events of all scales can be observed.

SOC theory has been a source of inspiration for metaheuristics and unconventional computing techniques. Extremal Optimization (EO) (Boettcher \& Percurs, 2003), for example, is based in the Bak-Sneppen model. EO uses a single solution vector that is modified by local search. The algorithm removes the worst components of the vector and replaces them with randomly generated material. By plotting the fitness of the solution, it is possible to observe distinct stages

Peer] Comput. Sci. reviewing PDF | (CS-2018:12:33477:1:1:NEW 23 Apr 2019) 
154 of evolution, where improvement is disturbed by brief periods of dramatic decrease in the 155 quality.

156 Lovbjerg and Krink (2002) modeled SOC in a PSO in order to control the convergence of the

157

158

159

160

161

162

163

164

165

166

167

168

169

170

171

172

173

174

175

176

177

178

179

180

181

182

183

184

185

186

187

188

189

190

191

192

algorithm and maintain population diversity. The authors claim that their method is faster and attains better solutions than the standard PSO. However, the algorithm adds several parameters to the standard PSO parameter set : overall five parameters must be tuned or set to constant $a d$ hoc values.

Complex and dynamic population structures have been one of most popular PSO research areas in the last decade. The comprehensive-learning PSO (CLPSO) (Liang et al., 2006; Lynn \& Suganthan, 2015) abandons the global best information, replacing it by a complex and dynamic scheme that uses all other particles' past best information. The algorithm significantly improves the performance of other PSOs on multimodal problems.

(Ratnaweera et al. 2004) propose new parameter automation strategies that act upon several working mechanisms of the algorithm. The authors introduce the concepts of time-varying acceleration coefficients (PSO-TVAC) and also mutation, by adding perturbations to randomly selected modulus of the velocity vector. Finally, the authors describe a self-organizing hierarchical particle swarm optimizer with time-varying acceleration coefficients (HPSOTVAC), which restricts the velocity update policy to the influence of the cognitive and social part, reinitializing the particles whenever they are stagnated in the search space.

(Liu et al., 2014) describe a PSO that uses a scale-free (SF) network for connecting the individuals. SF-PSO attains a better balance between solution quality and convergence speed when compared to standard PSOs with gbest and lbest neighborhood topology. However, the algorithm is not compared under more sophisticated frameworks or against state-of-the art PSOs, Furthermore, the size of the test set is small and does not comprise shifted or rotated functions.

Finally, (Vora \& Mirlanalinee, 2017) propose a dynamic small world PSO (DWPSO). Each particle communicates with the four individuals of its von Neumann neighborhood, to which two random connections are added (and then removed) in each time step. In other words, the neighborhood of each particle is comprised of six particles, four of them fixed throughout the run while the remaining two keep changing. The authors compare the performance of DSWPSO with other PSOs and conclude that due to a more balanced exploration and exploitation trade-off, DSWPSO is consistently better.In this work, the Bak-Sneppen model is used to design an alternative update strategy for the PSO. The strategy has been previously tested on a set of benchmark functions and compared to a standard S-PSO (Fernandes et al. 2016). The results show that SS-PSO significantly improves the performance of a S-PSO structured in a 2dimensional square lattice with Moore neighborhood. This paper is an extension of the aforementioned work. The main contributions here are: a) a complete statistical analysis of the performance, comparing the algorithm with standard PSOs and variations of the proposed strategy; b) a parameter sensitivity analysis and scalability tests showing that the performance enhancement introduced by the steady-state strategy is maintained throughout a reasonable range

Peer) Comput. Sci. reviewing PDF | (CS-2018:12:33477:1:1:NEW 23 Apr 2019) 
193 of parameter values and search space dimension ranging from 10 to 50; and, c) a comparison with state-of-the-art dynamic PSOs: CLPSO, PSO-TVAC and DSWPSO..

195

196

\section{Materials \& Methods}

197

198

199

200

201

202

203

204

205

206

207

\section{SS-PSO algorithm}

SS-PSO was inspired by a similarity between PSO and the Bak-Sneppen model: both are population models in which the individuals are structured by a network and evolve towards better fitness values. With this likeness in mind, we have devised an asynchronous and steady state update strategy for PSO in which only the least fit particle and its neighbors are updated and evaluated in each time step. Please note that SS-PSO is not an extinction model like the BakSneppen system: the worst particle and its neighbors are not replaced by random values; they are updated according to Equations 1 and 2. As for the other particles, they remain steady - hence the name of the algorithm: Steady-State Particle Swarm Optimization.

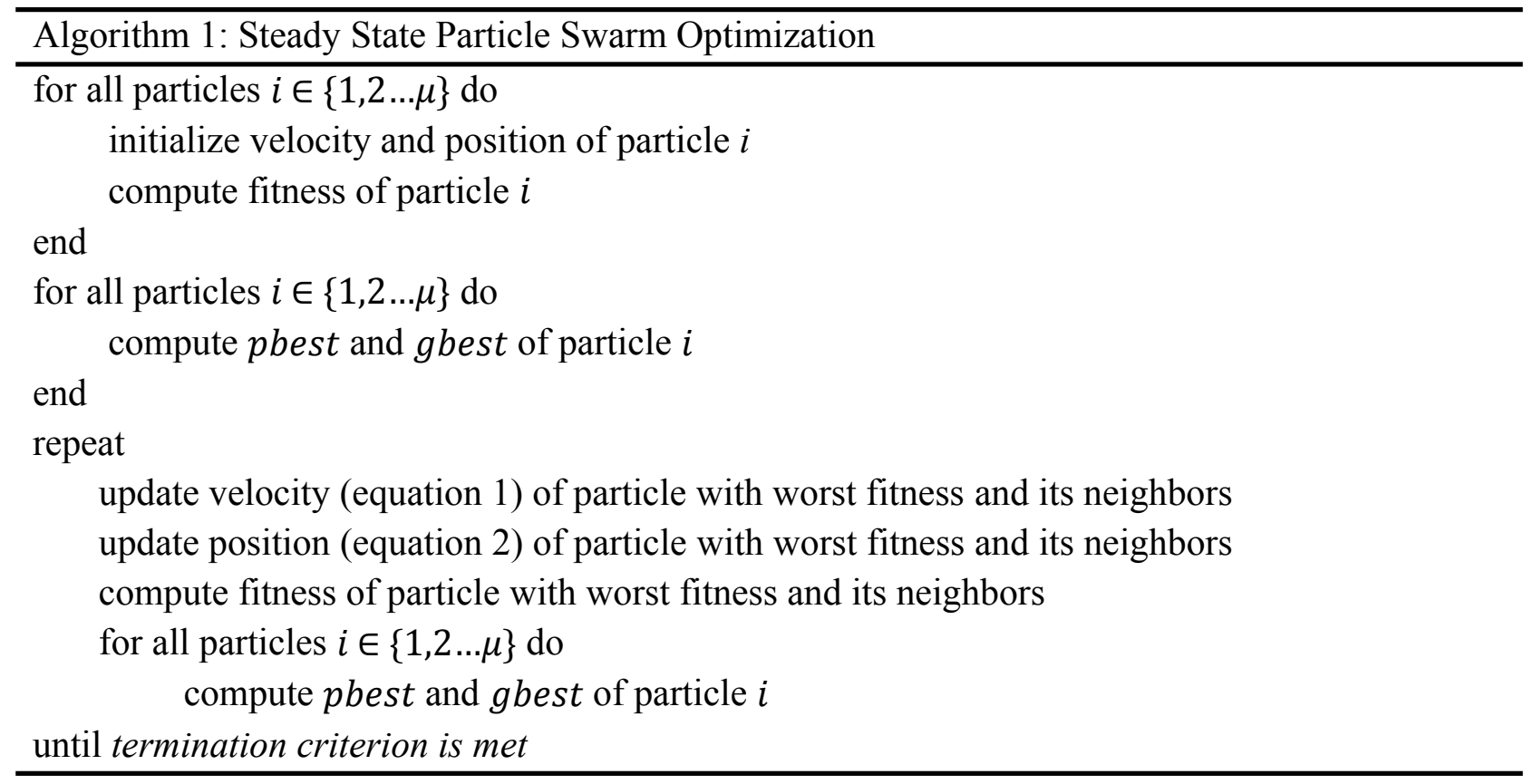

208

209

210

211

212

213

214

215

216

217

The particles to be updated are defined by the social structure. For instance, if the particles are connected by a lbest topology with $k=3$, then only the worst particle and its two nearest neighbors are updated and evaluated. Please note that local synchronicity is used here: the fitness values of the worst and its neighbors are first computed and only then the particles update their velocity. For the remaining mechanisms and parameters, the algorithm is exactly as a standard PSO. For a detailed description of SS-PSO, please refer to Algorithm 1.

The PSOs discussed in this paper, including the proposed SS-PSO, are available in the OpenPSO package, which offers an efficient, modular and multicore-aware framework for experimenting with different approaches. OpenPSO is composed of three modules: 
218 1. A PSO algorithm library.

2192 2. A library of benchmarking functions.

220 3. A command-line tool for directly experimenting with the different PSO algorithms and 221 benchmarking functions.

222 The library components can be interfaced with other programs and programming languages, 223 making OpenPSO a flexible and adaptable framework for PSO research. Its source code is

\section{Experimental Setup}

228

229

230

231

232

For testing the algorithm, ten benchmark problems (Table 1) are used. Functions $f_{1}-f_{3}$ are unimodal; $f_{4}-f_{8}$ are multimodal; $f_{9}$ is the shifted $f_{2}$ with noise and $f_{10}$ is the rotated $f_{5}\left(f_{9}\right.$ global optimum and $f_{10}$ matrix were taken from the CEC2005 benchmark). Population size $\mu$ is set to 49 . This particular value, which lies within the typical range (Kennedy \& Eberhart, 1995), was set in order to construct square lattices with von Neumann and Moore neighborhood. Following (Rada-Vilela et al., 2013), $c_{1}$ and $c_{2}$ were set to 1.494 and $\omega$ to 0.7298 . Xmax, the maximum

233

234

235

236

237

238

239

240

241

242

243

244

245

246

247

248

249

250

251

252

253

254

255

256

257 position value, and $V \max$, the maximum velocity value, are defined by the domain's upper limit. Asymmetrical initialization is used, with the initialization ranges in Table 1. Each algorithm was executed 50 times with each function and statistical measures were taken over those 50 runs. Stop criteria have been defined according to the functions and objectives of the experiments (see details in the Results section).

This work reports an extensive study of the proposed methodology. Different kinds of experiments have been performed, each one to investigate different aspects of the steady-state update strategy. The first experiment attempts at a proof-of-concept: SS-PSO is compared with standard (and synchronous) update strategies. The objective of the second experiment is to check if the convergence speed-up is caused indeed by the selective strategy or instead by the restricted evaluation pool, which is a consequence of the proposed method. The third test aims at studying the parameter sensitivity and the scalability with problem size. For that purpose, several tests have been conducted in a wide range of parameter values and problem dimension. The fourth experiment investigates SS-PSO under time-varying parameters and experiment number five compares SS-PSO with dynamically structured PSOs.

\section{Results}

\section{Proof-of-concept}

The first experiment intends to determine if SS-PSO is able to improve the performance of a standard S-PSO. For that purpose, three S-PSOs with different topologies have been implemented: lbest with $k=3$ (or ring) and 2-dimensional square lattices with von Neumann ( $k$ $=5)$ and Moore neighborhood $(k=9)$. Gbest $(k=n)$ is not included in the comparisons because SS-PSO uses the neighborhood structure to decide how many and which particles to update: for instance, in the von Neumann topology $(k=5)$, five particles are updated. Since gbest has $k=n$, the proposed strategy would update the entire population, i.e., it would be 
258

259

260

261

262

263

264

265

266

267

268

269

270

271

272

273

274

275

276

277

278

279

280

281

282

283

284

285

286

287

288

289

290

291

292

293

294

295

equivalent to a S-PSO. Therefore, we have restricted the study to lbest, von Neumann and Moore structures, labeling the algorithms, respectively, S-PSO ${ }_{\mathrm{lbest}}, \mathrm{S}-\mathrm{PSO}_{\mathrm{VN}}$ and S-PSO $\mathrm{Poore}_{\text {. }}$

Two sets of experiments were conducted. First, the algorithms were run for a specific amount of function evaluations (49000 for $f_{1}, f_{3}$ and $f_{6}, 980000$ for the remaining). After each run, the best solution was recorded. In the second set of experiments the algorithms were all run for 980000 function evaluations or until reaching a function-specific stop criterion (given in Table 1). A success measure was defined as the number of runs in which an algorithm attains the stop criterion. This experimental setup is similar to those in (Kennedy \& Mendes, 2002) and (RadaVilela et al., 2013). The dimension of the functions search space is $D=30$ (except $f_{6}$, with $D=2$ ). The results are in Table 2 (fitness), Table 3 (evaluations) and Table 4 (success rates). The best results among the three algorithms are shown in bold.

When compared to $\mathrm{S}-\mathrm{PSO}_{\mathrm{lbest}}$, S-PSO $\mathrm{P}_{\text {Moore }}$ attains better solutions (considering median values of fitness distributions over 50 runs) in most of the functions and is faster (considering median values of evaluations required to meet the criteria) in every function. When compared to S$\mathrm{PSO}_{\mathrm{VN}}, \mathrm{S}^{\mathrm{PSO}} \mathrm{PMore}_{\mathrm{M}}$ is faster in every function and yields better median fitness values in unimodal functions.

In terms of success rates, S-PSO ${ }_{\text {Moore }}$ clearly outperforms the other topologies in function $\boldsymbol{f}_{\mathbf{9}}$ and is much more efficient than S-PSO ${ }_{\text {lbest }}$ in function $\boldsymbol{f}_{4}$. These results are consistent with (Kennedy \& Mendes, 2002).

The algorithms were ranked by the Friedman test for each function. Table 5 shows the ranks according to the quality of solutions, while Table 6 shows the ranks according to the convergence speed (only the functions on which the three algorithms attained the same success

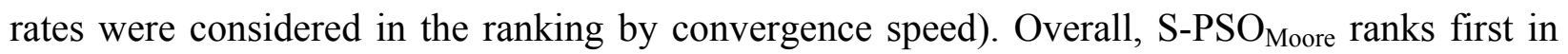
terms of solutions quality and convergence speed - see Fig. 1. Therefore, we conclude that the Moore structure is well suited for assessing the validity and relevance the SS-PSO.

Once the best network has been found for this particular set of problems, the next step was to compare synchronous and asynchronous PSOs on the most efficient topology. For that purpose, we have implemented a SS-PSO ${ }_{\text {Moore }}$ and tested it on the 10-function set under the same conditions described above. The results can be found in Table 7.

Table 8 gives a comparison between the performance of S-PSO $\mathrm{Moore}_{\text {and }} \mathrm{SS}-\mathrm{PSO}_{\text {Moore }}$ based on the numerical results and statistical analysis of those same results. The non-parametric MannWhitney test was used to compare the distribution of fitness values and number of evaluations to meet criteria of each algorithm in each function. The ranking of fitness distributions are significant at $p \leq 0.05$ for $f_{1}, f_{2}, f_{3}, f_{6}, f_{7}, f_{9}$, i.e., in these functions, the null hypothesis that the two samples come from the same population is rejected. For the remaining functions $\left(f_{5}, f_{8}\right.$ $f_{10}$, the null hypothesis is not rejected: the differences are not significant.

In terms of function evaluations, SS-PSO ${ }_{\text {Moore }}$ is faster in the entire set of unimodal problems. In multimodal problems, $\mathrm{SS}^{\mathrm{PSO}} \mathrm{Moore}_{\mathrm{M}}$ needs less evaluations in $f_{5}, f_{6}, f_{7}$ and $f_{8}$. Results of 
296

297

298

299

300

301

302

303

304

305

306

307

308

309

310

311

312

313

314

315

316

317

318

319

320

321

322

323

324

325

326

327

328

329

330

331

332

333

334

Mann-Whitney tests are significant at $p \leq 0.05$ for functions $f_{1}, f_{2}, f_{3}, f_{5}, f_{7}, f_{8}, f_{9}$ and $f_{10}-$ see Table 8.

The success rates are similar, except for $f_{7}$ (in which SS-PSO clearly outperforms the standard algorithm) and $f_{9}$. In conclusion: empirical results, together with statistical tests, show that according to accuracy, speed and reliability, SS-PSO Moore $_{\text {outperforms S-PSO }}$ Moore in most of the benchmark functions selected for this test, while not being outperformed in any case.

\section{Update Strategy}

The preceding tests show that the steady state update strategy when implemented in a PSO structured in a lattice with Moore neighborhood improves its performance. The following experiment aims at answering an important question: what is the major factor in the performance enhancement? Is it the steady state update, or instead the particles that are updated?

In order to investigate this issue, two variants of SS-PSO were implemented: one that updates the best particle and its neighbors (replace-best); and another that updates a randomly selected particle and its neighbors (replace-random). The algorithms were tested on the same set of

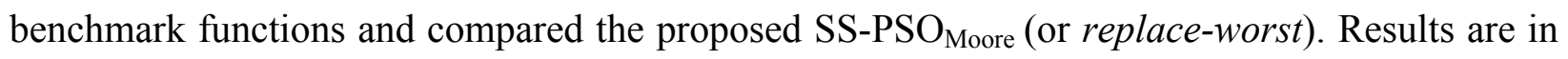

\section{Table 9.}

Replace-best update strategy is outperformed by replace-worst SS-PSO. With the exception of $f_{1}$ and $f_{3}$, the quality of solutions is degraded when compared to the proposed SS-PSO. However, success rates are considerably lower in most functions, including $f_{1}$ and $f_{3}$. Please note that functions $f_{1}$ and $f_{3}$ are unimodal and therefore they can be easily solved by hillclimbing and greedy algorithms. It is not surprising that a greedy selective strategy like SS-PSO with replace-best can find very good solutions in some runs. However, for more difficult problem, replace-best is clearly unable to find good solutions.

As for replace-random, it improves S-PSO in some functions, but in general is not better than replace-worst: replace-random SS-PSO is less accurate and slower in most of the functions. The Friedman test shows that SS-PSO with replace-worst strategy ranks first in terms of solutions quality - see Fig. 2.

Table 10 compares replace-random and replace-worst with the assistance of Mann-Whitney statistical tests. Except for $f_{4}$, replace-worst is significantly more efficient than replace-random. The experiment demonstrates that selective pressure imposed on the least fit individuals is the major factor in the performance of SS-PSO.

\section{Scalability}

The proof-of-concept showed that SS-PSO outperforms S-PSO in most of the functions in the test set, and the previous experiment demonstrate that the major factor in the performance enhancement is the pressure on the least fit particles. However, only instances of the problems with $D=30$ have been tested; therefore, another question arises at this point: does the improvement shown by SS-PSO hold for a wide range of problem sizes? In order to answer that 
335

336

337

338

339

340

341

342

343

344

345

346

347

348

349

350

351

352

353

354

355

356

357

358

359

360

361

362

363

364

365

366

367

368

369

370

371

372

373

374

question, we have conducted a scalability study: the algorithms were tested on the same set functions but with $\mathrm{D}$ ranging from 10 to 50 (except $f_{6}$, which is a 2-dimensional function and for that reason was excluded from this test).

As in previous experiments, the algorithms were first run for a limited amount of function evaluations and the best fitness values were recorded. Then, the algorithms were all run for 980000 evaluations or until reaching a function-specific stop criterion. The number of iterations required to meet the criterion was recorded and statistical measures were taken over 50 runs. (Function $f_{10}$ has not been tested for dimensions 20 and 40 because the CEC2005 benchmark, from where the orthogonal rotational matrices $\boldsymbol{M}$ have been taken, does not provide the matrices for those dimensions.)

Table 11 shows the median best fitness values attained by each algorithm on each instance of the problems and Table 12 shows the success rates. In terms of quality of solutions, the performance patterns observed with $D=30$ are maintained: the strategy does not introduce scalability difficulties. As for the success rates, except for a few instances, SS-PSO attains better or equal success rates.

The convergence speed has been graphically represented for better assessment of the effects of growing problem size - see Fig. 3. The graphs show that the proposed strategy does not introduce scalability difficulties (other than the ones intrinsic to standard PSOs). It also shows that, in general, SS-PSO is faster than S-PSO.

\section{Parameter Sensitivity}

PSO performance can be severely affected by the parameter values. The inertia weight and acceleration coefficients must be tuned in order to balance exploration and exploitation: if far from the optimal values, convergence speed and/or solution quality can be significantly reduced. Population size also influences the performance of population-based metaheuristics: larger populations help to maintain diversity, but they slow down convergence speed; on the other hand, smaller populations are faster but they are more likely to converge to local optima.

Furthermore, PSOs empirical studies usually depend on a single set of parameters for several functions with different characteristics. This is the case of this paper, in which a typical parameter setting has been used for evaluating the performance of the PSOs. That set of parameter is not expected to be the optimal tuning for every function, but instead a compromised solution to avoid the exponential growth of experimental procedures.

For these reasons, when testing a new PSO, it is important to investigate its sensitivity to the parameter values. With that purpose in mind, the following experimental procedure has been designed.

S-PSO and SS-PSO were tested on function $f_{1}$ (unimodal), $f_{2}$ (multimodal), $f_{9}$ (shifted and noisy) and $f_{10}$ (rotated) with the following parameter values: inertia weight was set to 0.6798 , $0.7048,0.7298,0.7548$ and 0.7798 , while acceleration coefficients and population size remained fixed at 1.494 and 49 ; then, $c_{1}$ and $c_{2}$ were set to $1.294,1.394,1.494,1.594$ and 1.694 while $\omega$ and $\mu$ remained fixed at 0.7298 and 49 , respectively; finally, population size was set to 36,49 
375

376

377

378

379

380

381

382

383

384

385

386

387

388

389

390

391

392

393

394

395

396

397

398

399

400

401

402

403

404

405

406

407

408

409

410

411

412

and 64 , while $\omega$ and the acceleration coefficients were set to 0.7298 and 1.4962 . The results are depicted in Figures 4-7.

The graphics show that the performance indeed varies with the parameter values, as expected. In the case of function $f_{1}$, other parameter settings attain better results than the ones used in previous section. However, the relative performance of S-PSO and SS-PSO maintains throughout the parameters ranges. In functions $f_{8}, f_{9}$ and $f_{10}$, the quality of solutions is in general maximized by $\omega$ and $\mathrm{c}$ values around the ones used in previous sections. Convergence speed, in general, improves with lower $\omega$, $\mathrm{c}$ and $\mu$ values.

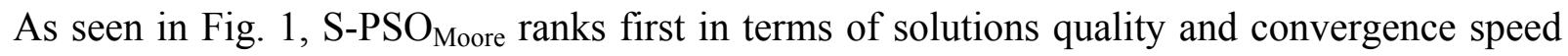
when compared to ring and von Neumann topologies. Although not a parameter in the strict sense of the term, the network topology is a design choice that significantly affects the performance of the algorithm: (Kennedy \& Mendes, 2002) investigated several types of networks and recommend the use of von Neumann lattices; (Fernandes et al., 2018) tested regular graphs and concluded that convergence speed improves with the degree of connectivity but success rates are in general degraded when $k$ is above 9 (equivalent to Moore neighborhood) and a that good compromise is achieved with $5 \leq k \leq 13$.

In order to study the performance of SS-PSO with different network topologies, regular graphs have been constructed with the following procedure: starting from a ring structure with $k=3$, the degree is increased by linking each individual to its neighbors' neighbors, creating a set of regular graphs with $k=\{3,5,7,9,11 \ldots, n\}$, as exemplified in Fig. 8 for population size 7. Parameters $c_{1}$ and $c_{2}$ were set to 1.494 and $\omega$ to 0.7298 and population size $\mu$ was set to 33 . The algorithms were all run for 660000 function evaluations or until reaching the function-specific stop criterion given in Table 1. Each algorithm has been executed 50 times with each function and statistical measures were taken over those 50 runs.

Fig. 9 shows the success rates and convergence speed of SS-PSO structured by topologies with varying $k$. Convergence increases with $k$, and achieves optimal values $13 \leq k \leq 25$ in most of the functions. However, as seen in Fig. 9(a), best success rates are achieved when $7 \leq k \leq 13$ (except $f_{10}$, for which $k=5$ is the best topology). These conclusions are similar to those by (Fernandes et al., 2018) related to the standard PSO and are coincident with the typical rule of thumb for PSOs: highly connected topologies are faster but less reliable, while topologies with lower connectivity require more evaluations to meet the convergence criteria but converge more often to the solution.

Please remember that we are not trying to find the best set of parameters for each function. The most important conclusions here is that SS-PSO does not seem to be more sensitive to the parameters than S-PSO, displaying similar patterns when varying $\omega, c_{1}$ and $c_{2}$ and $\mu$, and that the performance enhancement brought by SS-PSO is observed on a reasonably wide range of parameter values.

PeerJ Comput. Sci. reviewing PDF | (CS-2018:12:33477:1:1:NEW 23 Apr 2019) 


\section{Time-varying parameters}

414 An alternative approach to parameter tuning is to let the parameters values change during the 415 run, according to deterministic or adaptive rules. In order to avoid tuning effort and adapt the 416 balance between local and global search to the search stage, Shi and Eberhart (1999) proposed a 417 linearly time-varying inertia weight: starting with an initial and pre-defined value, the parameter 418 value decreases linearly with time, until it reaches the minimum value. The variation rule is 419 given by Equation (3):

420

$$
\omega(t)=\left(\omega_{1}-\omega_{2}\right) \times \frac{\left(\max \_t-t\right)}{\max _{-} t}+\omega_{2}
$$

421

422

428

429

430

where $t$ is the current iteration, max_t is the maximum number of iterations, $\omega_{1}$ the inertia weigh initial value and $\omega_{2}$ its final value.

Later, Ratnaweera et al. (2004) tried to improve Shi and Eberhart's PSO with time-varying inertia weight (PSO-TVIW) using a similar concept applied to the acceleration coefficients. In the PSO with time-varying acceleration coefficients PSO (PSO-TVAC) the parameters $\mathrm{c}_{1}$ and $\mathrm{c}_{2}$ change during the run according to the following equations:

$$
\begin{aligned}
& c_{1}=\left(c_{1 f}-c_{1 i}\right) \times \frac{t}{\max \_t_{t}}+c_{1 i} \\
& c_{2}=\left(c_{2 f}-c_{2 i}\right) \times \frac{t}{\max \_t}+c_{2 i}
\end{aligned}
$$

where $c_{1 i}, c_{1 f}, c_{2 i}, c_{2 f}$ are the acceleration coefficients initial and final values.

The experiments in this section compare PSO-TVAC with SS-PSO-TVAC (i.e., PSO-TVAC with the steady-state update strategy). Parameters $\omega_{1}$ and $\omega_{2}$ were set to 0.75 and 0.5 . The acceleration coefficient $c_{1}$ initial and final values were set to 2.5 and 0.5 and $c_{2}$ ranges from 0.5 to 2.5, as suggested by Ratnaweera et al. (2004). The results are in Table 13 (PSO-TVAC) and Table 14 (SS-PSO-TVAC).

Table 15 compares the algorithms using Mann-Whitney tests. SS-PSO-TVAC improves PSOTVAC in every unimodal function in terms of accuracy and convergence speed and it is significantly faster in functions $\mathrm{f}_{6}, \mathrm{f}_{7}, \mathrm{f}_{8}$ and $\mathrm{f}_{10}$ while attaining similar results. PSO-TVAC only outperforms SS-PSO-TVAC in the noisy $\mathrm{f}_{9}$ function. These results show that the steady state version of PSO-TVAC is able to improve the convergence speed of the original algorithm in several types of fitness landscapes. Furthermore, SS-PSO-TVAC achieves more accurate solutions in the unimodal problems. 


\section{Comprehensive Learning PSO}

445 The following experiment aims at comparing the proposed SS-PSO with the comprehensive 446 learning PSO (CLPSO) (Liang et al., 2006; Lynn \& Suganthan, 2015). CLPSO uses an 447 alternative velocity updating equation:

448

449

450

451

452

453

454

455

456

457

458

459

460

461

462

463

464

465

466

467

468

469

470

471

472

473

474

475

476

477

478

479

480

$$
v_{i, d}(t)=\omega \times v_{i, d}(t-1)+c \times r \times\left(p_{f i(d), d}-x_{i, d}(t-1)\right)
$$

where $\overrightarrow{f_{i}}=\left(f_{i}(1), f_{i}(2), \ldots f_{i}(D)\right)$ defines which particle's best solutions particle $i$ should follow. Hence, the term $p_{f i(d), d}$ can refer to the corresponding dimension of any particle's best found solution so far. The decision depends on a probability $p_{c}$, different for each particle and computed a priori. Following the guidelines and parameters in (Liang et al., 2006), CLPSO and SS-CLPSO have been implemented and tested in the set of 10 benchmark functions.

CLPSO performance is strongly dependent on the refreshing gap parameter $m$, which defines the number of generations during which the particles are allowed to learn from $f_{i}$ without improving their fitness. After $m$ generations without fitness improvement, $f_{i}$ is reassigned. In order to make fair comparisons, parameter $m$ was first optimized for each function. The other parameters were set as in (Liang et al., 2006). Then, SS-CLPSO was tuned using the same parameter setting as the corresponding CLPSO.

The results are in Table 16 and 17 and statistical analysis is in Table 18. On the one hand, the results show that, in general, a steady-state strategy applied to CLPSO does not improve the performance of the algorithm. On the other hand, SS-CLPSO does not degrade the general behavior of CLPSO. Please note that CLPSO does not use a traditional topology. In this case, to construct SS-CLPSO, we use a Moore neighborhood to decide which particles to update along with the least fit individuals, but, unlike SS-PSO or SS-PSO-TVAC, the structure does not define the information flow within the swarm. Since neighboring particles communicate and use each other's information, they tend to travel through similar regions of the landscape, but in CLPOS there is not necessarily a relationship between the particles in the set and this clustering behavior is not present. For a steady-state strategy to take full advantage of the CLPSO dynamic network, maybe it is necessary to define a dynamic update strategy takes into account the current set of particles from which an individual is learning at a specific period of the run. Steady-state updates strategies for PSO in dynamic networks is planned as future work.

\section{Dynamic Small World PSO}

The final experiment compares SS-PSO with the Dynamic Small World PSO (DSWPSO), recently proposed by Vora \& Mirnalinee (2017). DSWPSO uses a static von Neumann topology to which a number of random connections are added in each iteration. It is a very simple variation of the standard PSO, but it attains quite interesting results when compared to a number of state-of-the-art PSOs. 
481

482

483

484

485

486

487

488

489

490

491

492

493

494

495

496

497

498

499

500

501

502

503

504

505

506

507

508

509

510

511

512

513

514

515

516

517

518

519

520

For this paper, DSWPSO was tested with von Neumann and Moore topologies. The number of random neighbors in each topology was set to 2, as suggested by (Vora \& Mirnalinee, 2017). Parameters $c_{1}$ and $c_{2}$ were set to 1.494 and $\omega$ to 0.7298 . The algorithms were all run for 200000 function evaluations. DSWPSO results are presented in Tables 19 (von Neumann) and 20 (Moore). The statistical analysis that compares SS-PSO and DSWPSO are in Tables 21 (von Neumann) and 22 (Moore). It is clear that SS-PSO outperforms DSWPSO with both von Neumann and Moore base-topology in most of the functions, not only in terms of convergence speed, but also in solution quality.

Figure 10 shows the convergence curves (median best fitness values over 50 runs) of S-PSO, SS-PSO and DSWPSO (von Neumann). The graphics show that SS-PSO converges faster to the vicinity of the solutions. Furthermore, and although it is not perceivable in the graphics, SS-PSO eventually reaches solutions closer to $f(x)=0$ (the optimum of both functions) as demonstrated by Tables 8 and 21 ,

\section{Running times}

A final experiment compares S-PSO and SS-PSO running times. The algorithms are run on function $f_{7}$ with $D$ set to $10,30,50$ and 100. Moore neighborhood is used in both algorithms and parameters are set as in previous experiments. Fig. 11 shows the running times of 49000 functions evaluations (median values over 10 runs for each algorithm). The running times of each algorithm are statistically equivalent for every $D$ value. Running times of SS-PSO with von Neumann and Moore neighborhood are also equivalent. The PerfAndPubTools software (Fachada et al., 2016) was used to analyze the running times.

\section{Discussion}

The experiments in the previous sections demonstrate that SS-PSO is able to significantly improve the performance of the standard PSO, at least on the set of benchmark functions. The differences are particularly noticeable in the convergence speed of the algorithms, but SS-PSO is also able to improve the solution quality in several functions (see Table 8). An experiment comparing three different steady-state strategies show that replacing the worst particle and its neighbors is the best strategy. Our initial hypothesis (reducing the number of evaluations in each time step, while focusing only on the worst solutions, reduces the computational effort to reach a solution) is confirmed.

The relative performance of SS-PSO and standard PSO has also been verified for a wide range of parameter values (see Figures 4-7) as well as for different problem dimensions (see Figure 3). These results are important since they demonstrate that the proposed strategy has not been fine-tuned and that its validity is not restricted to a particular region of the parameter space or problem dimension. The algorithm was also compared to a PSO with time-varying acceleration, 
521 again attaining good results, thus reinforcing the idea that the steady-state strategy is consistent 522 and robust. SS-PSO was compared to CLPSO, and while being outperformed in terms of solution 523 quality in four functions, it yields better solutions in two problems, and is faster in other two 524 functions. Since CLPSO is considered to be a very efficient algorithm, these results are 525 promising. It deserves further examination whether variants of SS-PSO could clearly outperform

526

527

528

529

530

531

532

533

534

535

536

537

538

539

540

541

542

543

544

545

546

547

548

549

550

551

552

553

554

555

556

557

558

559

560

CLPSO. Finally, SS-PSO was compared to DSWPSO with excellent results.

\section{Conclusions}

This paper investigates the performance of a new and unconventional updated strategy for the PSO. The steady state PSO (SS-PSO) is inspired by the Bak-Sneppen model of coevolution. However, while in the Bak-Sneppen model the worst individual and its neighbors are replaced by random values, in SS-PSO the worst particle and its neighbors are updated and evaluated in each time step. The remaining particles are kept in a steady state until they eventually satisfy the update criterion. Due to its strategy, SS-PSO may be classified within the asynchronous PSOs category. However, its working mechanisms are radically different from standard asynchronous PSOs.

After preliminary tests that determined the best topology for a set of ten unimodal, multimodal, shifted, noisy and rotated benchmark problems, the strategy was implemented on the winning structure: 2-dimensional lattice with Moore neighborhood. Quality of solutions, convergence speed and success rates were compared and statistical analyses were conducted on the results. SS-PSO significantly improved the performance of a standard synchronous PSO (SPSO) in every function, at least in one of the two criteria (quality of final solutions and convergence speed). A parameter sensitivity analysis showed that SS-PSO is not more sensitive to the variation of parameter values than S-PSO. A scalability test showed that the proposed strategy does not introduce scalability difficulties. The algorithm was compared to the PSO with time-varying acceleration coefficients (PSO-TVA) and to the comprehensive learning PSO (CLPSO) with good results.

The first step in future works is to increase the size of the test with more functions, hoping that an extended test set can improve our insight into the behavior of the algorithm. The emergent properties of the algorithm (size of events, duration of stasis, critical values) will be also studied and compared to those of the Bak-Sneppen model. Finally, steady-state update strategies in dynamic topologies will be investigated.

\section{References}

Allmendiger R., Li X., Branke J. 2008. Reference Point-Based Particle Swarm Optimization Using a Steady-State Approach, SEAL 2008. Lecture Notes in Computer Science, vol 5361, 200-209.

Aziz NAB, Mubin M, Mohamad MS, Aziz KA. 2014. A Synchronous-Asynchronous Particle Swarm Optimisation Algorithm, The Scientific World Journal, Volume 2014, Article ID 123019,

Peer) Comput. Sci. reviewing PDF | (CS-2018:12:33477:1:1:NEW 23 Apr 2019) 
56117 pages.Carlisle A, Dozier G. 2001. An off-the-shelf PSO. Workshop on Particle Swarm 562 Optimization.

563 Baillieul J, Samad T. 2015. Encyclopedia of Systems and Control, London: Springer-Verlag.

564 Bak P, Tang C, Wiesenfeld K. 1987. Self-organized Criticality: an Explanation of 1/f Noise, 565 Physical Review Letters, 59(4), 381-384.

566 Bak P, Chen K, Tang C. 1990. A forest-fire model and some thoughts on turbulence, Physics 567 Letters, A 147, 197-300.

568 Bak P, Sneppen K. 1993. Punctuated equilibrium and criticality in a simple model of evolution, $569 \quad$ Physical Review Letters, 71 (24), 4083-4086.'

570 Bak P. 1996. How Nature Works. New York: Springer-Verlag, 1996.

571 Boettcher S, Percus AG. 2003. Optimization with Extremal Dynamics, Complexity, 8(2), 57-62.

572 Dagum L, Menon R. 1998. OpenMP: an industry standard API for shared-memory 573 programming. IEEE computational science and engineering, 5(1), 46-55.

574 Engelbrecht AP. 2013. Particle Swarm Optimization: Iteration Strategies Revisited, 2013 BRICS

575 Congress on Computational Intelligence and 11th Brazilian Congress on Computational 576 Intelligence, 119-123.

577 Fachada N, Lopes V.V, Martins RC, Rosa AC. 2016. PerfAndPubTools - Tools for Software 578 Performance Analysis and Publishing of Results. Journal of Open Research Software, 4(1), 579 e18.

580 Fernandes CM, Laredo J.L.J., Merelo J.J., Cotta C., Rosa A.C. 2014. Particle Swarms with 581 Dynamic Topologies and Conservation of Function Evaluations. Proceedings of the 582 International Joint Conference on Computational Intelligence, 86-94.

583 Fernandes CM, Merelo JJ, Rosa AC. 2016. An Asynchronous and Steady State Update Strategy 584 for the Particle Swarm Optimization Algorithm, Proceedings of Parallel Problem Solving 585 from Nature - PPSN XIV, Springer, pp. 166-177.

586 Fernandes, C.M; Fachada, N.; Laredo, J.; Merelo, J.; Castillo, P. and Rosa, A.C. 2018. Revisiting

587

588

589

590

591

592

593

594

595

596

597

598

599

600 Population Structure and Particle Swarm Performance. Proceedings of the 10th International Joint Conference on Computational Intelligence - Volume 1: IJCCI, pp. 248-254

Gutenberg B, Richter, C. F. 1956. Magnitude and Energy of Earthquakes. Annali di Geofisica, 9, $1-15$.

Kennedy J, Eberhart R. 1995. Particle Swarm Optimization, Proceedings of IEEE International Conference on Neural Networks, Vol.4, 1942-1948.

Kennedy J, Mendes R. 2002. Population structure and particle swarm performance, Proceedings of the IEEE World Congress on Evolutionary Computation, 1671-1676.

Løvbjerg M, Krink T. 2002. Extending particle swarm optimizers with self-organized criticality, Proceedings of the 2002 IEEE Congress on Evolutionary Computation, Vol. 2, IEEE Computer Society, 1588-1593.

Liang JJ, Qin AK, Suganthan PN. 2006. Comprehensive Learning Particle Swarm Optimizer for Global Optimization of Multimodal Functions, IEEE Transactions on Evolutionary Computation, 10(3), 281-294. 
601 Liu C., Du W-B, Wang W-X. 2014. Particle Swarm Optimization with Scale-free Interactions,

602

603

604

605

606

607

608

609

610

611

612

613

614

615

616

617

618

619

620

621

622

$$
\text { PLoS ONE 9(5): e97822. doi:10.1371/journal.pone.0097822. }
$$

Lynn N, Suganthan PN. 2015. Heterogeneous comprehensive learning particle swarm optimization with enhanced exploration and exploitation, Swarm and Evolutionary Computation, 24, pp. 11-24.

Majercik S. 2013. GREEN-PSO: Conserving Function Evaluations in Particle Swarm Optimization, Proceedings of the IJCCI 2013, 160-167.

$\mathrm{McNabb}$ A. 2014. Serial PSO results are irrelevant in a multi-core parallel world, Proceedings of the 2014 IEEE Congress on Evolutionary Computation, 3143-3150.

Rada-Vilela J, Zhang M., Seah W. 2013. A Performance Study on Synchronous and Asynchrounous Updates in Particle Swarm, Soft Computing, 17(6), 1019-1030.

Ratnaweera, A., Halgamuge, S. \& Watson, H. (2004). Self-organizing hierarchical particle swarm optimizer with time varying accelerating coefficients, IEEE Transactions on Evolutionary Computation, 8(3), 240-255.

Shi Y, Eberhart R.C. 1999. Empirical study of particle swarm optimization, Proceedings of IEEE International Congress on Evolutionary Computation, vol. 3,101-106.

Vora M., Mirlanalinee T.T. 2017. Dynamic small world particle swarm optimizer for function Optimization, Natural Computing, 17, 901-917.

Whitley D, Kauth J 1988. GENITOR: A different Genetic Algorithm, Proc. of the Rocky Mountain Conf. on Artificial Intelligence.

Zhang C., Zhang Yi, 2011. Scale-free fully informed particle swarm optimization algorithm, Infornation Sciences, 181, 4550-4568.

PeerJ Comput. Sci. reviewing PDF | (CS-2018:12:33477:1:1:NEW 23 Apr 2019) 
Figure 1 (on next page)

S-PSO ${ }_{\text {lbest }}$ S-PSO ${ }_{v N}$ and $\mathrm{S}-\mathrm{PSO}_{\text {moore }}$ : Solutions quality and convergence speed rank by the Friedman test. 
PeerJ Computer Science

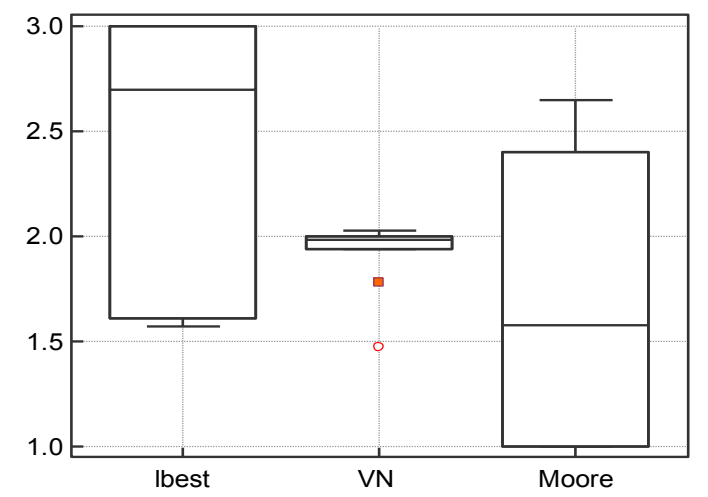

(A) fitness
Manuscript to be reviewed

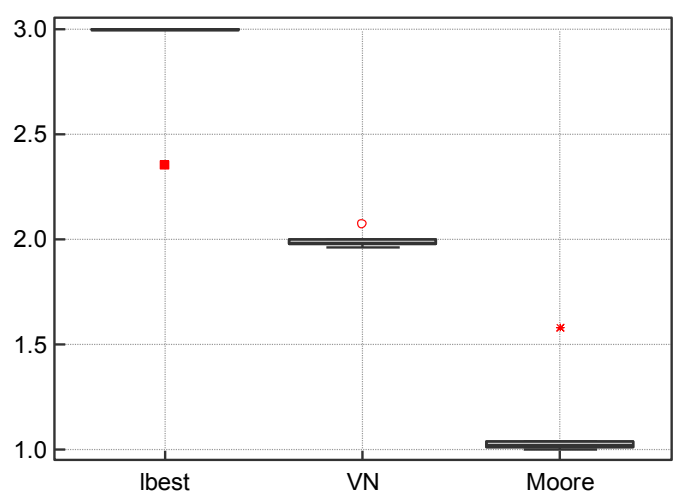

(B) convergence speed 
Figure 2 (on next page)

Fitness rank by Friedman test. 


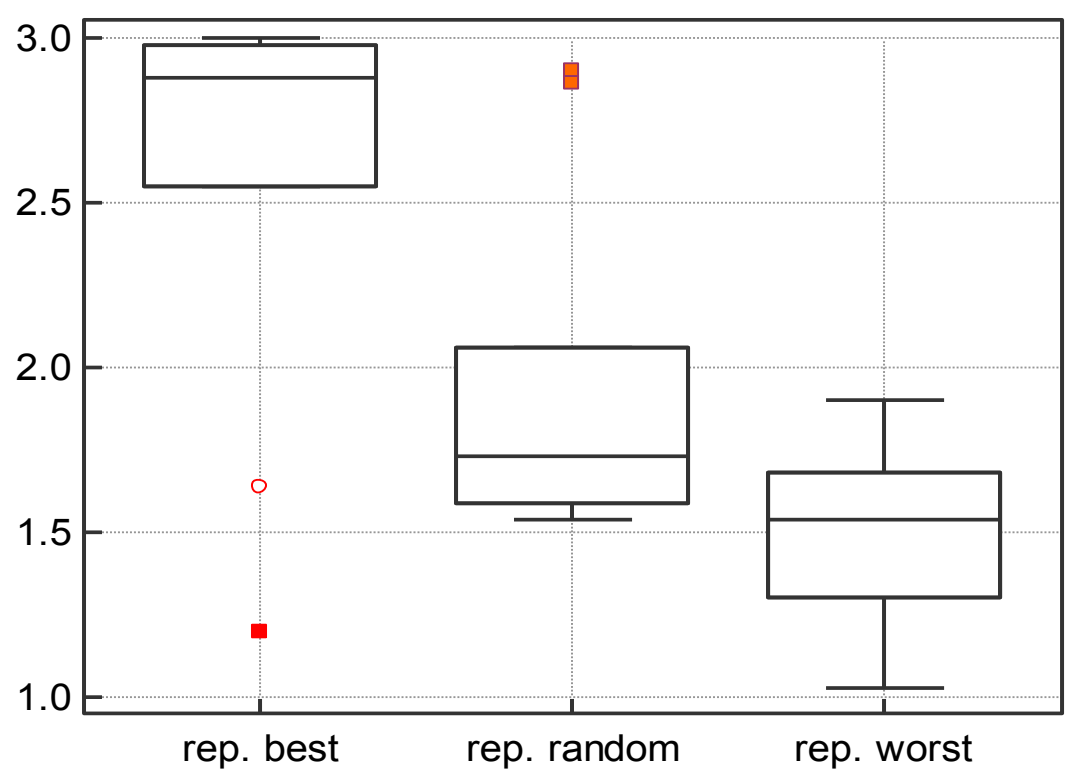


Figure 3 (on next page)

Convergence speed and problem dimension. 
PeerJ Computer Science

(A) Sphere

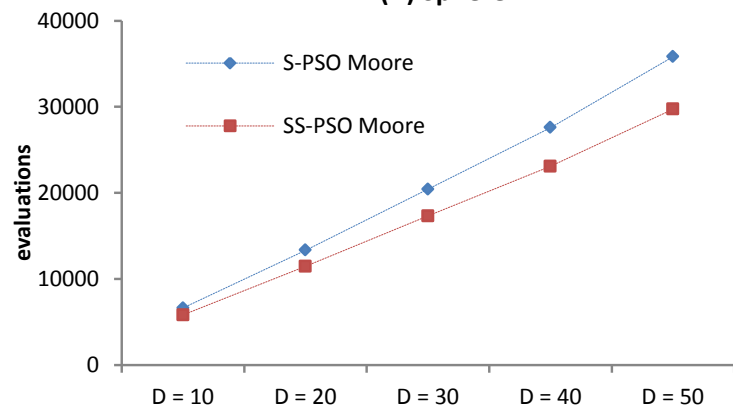

(C) Hyper

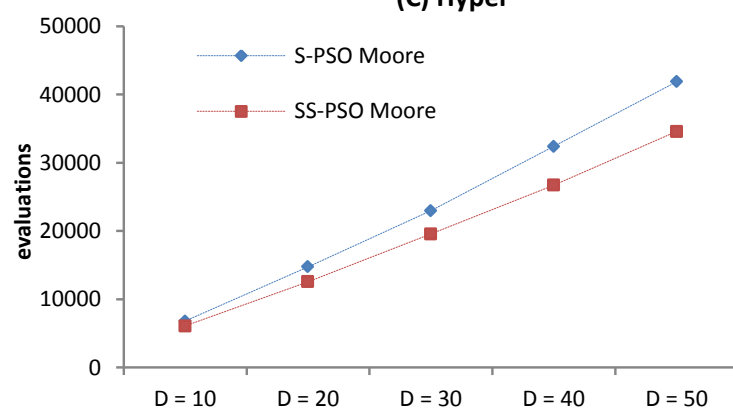

(E) Griewank

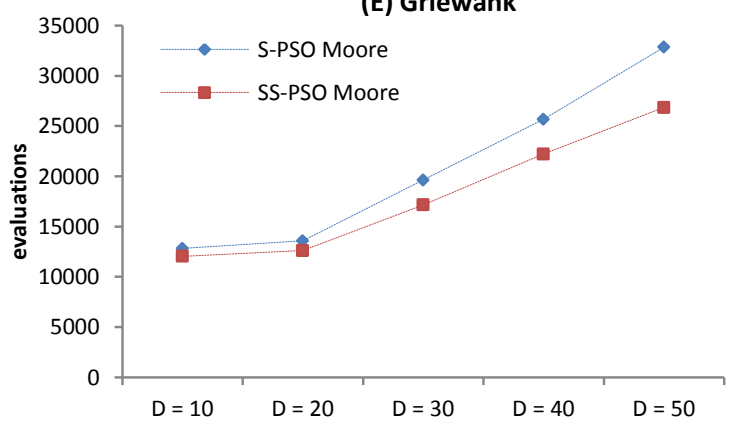

(G) Ackley

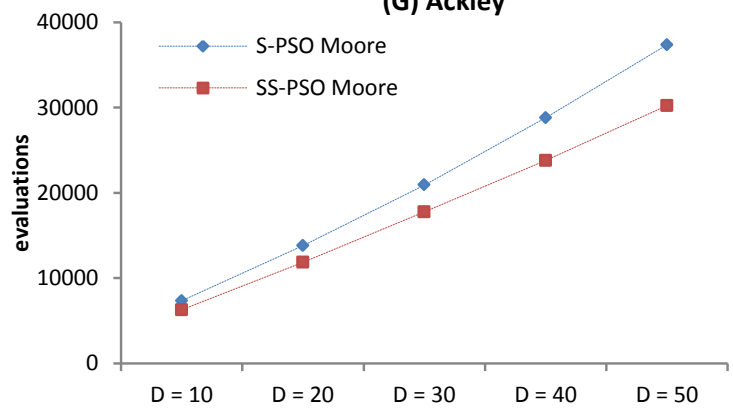

(I) Rotated Griewank

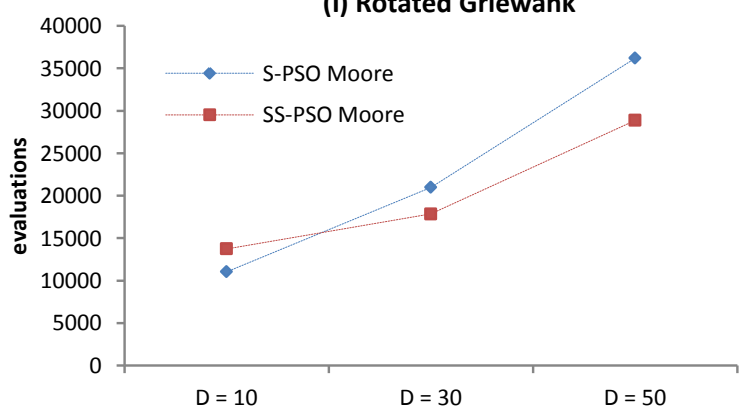

\section{Manuscript to be reviewed}

(B) Quadric

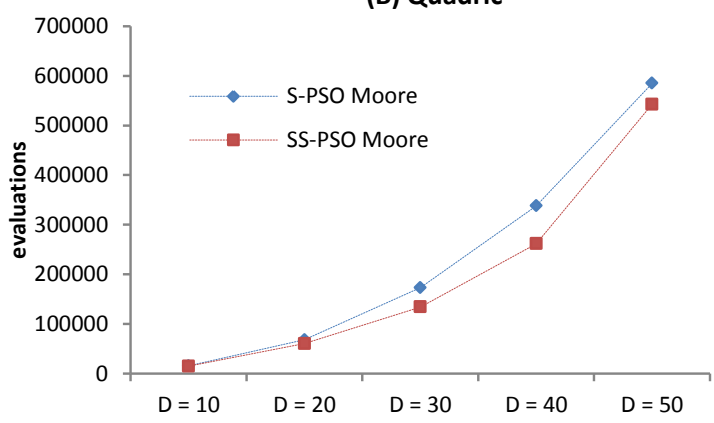

(D) Rastrigin

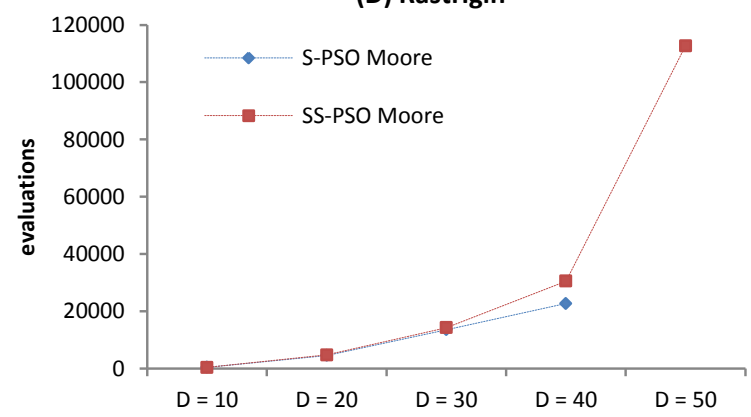

(F) Weierstrass

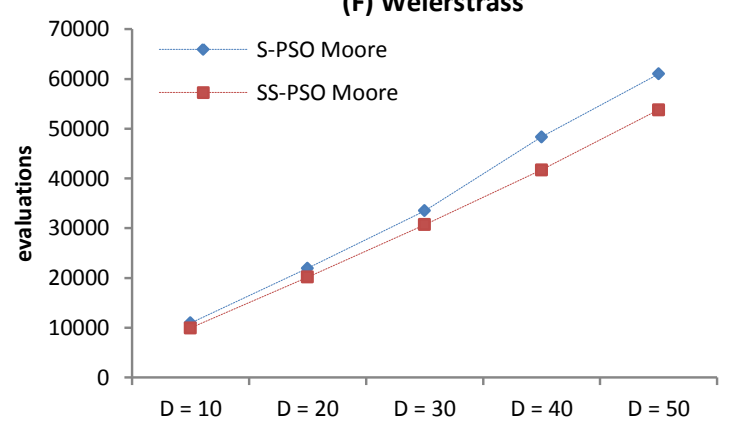

(H) Shifted Quadric with noise

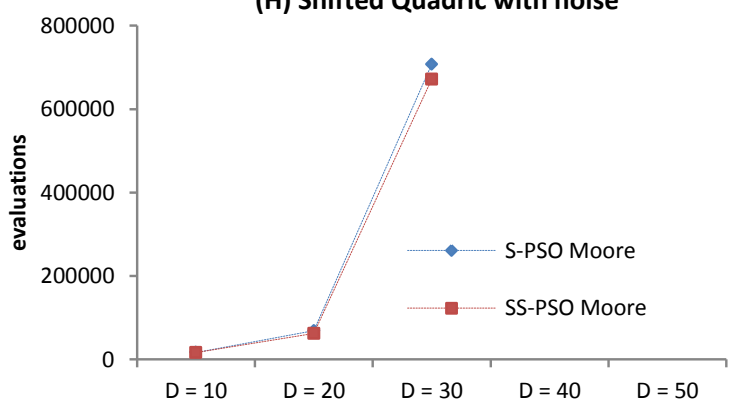


Figure 4 (on next page)

Parameters sensitivity: Sphere function (f1). 
PeerJ Computer Science

\section{A}

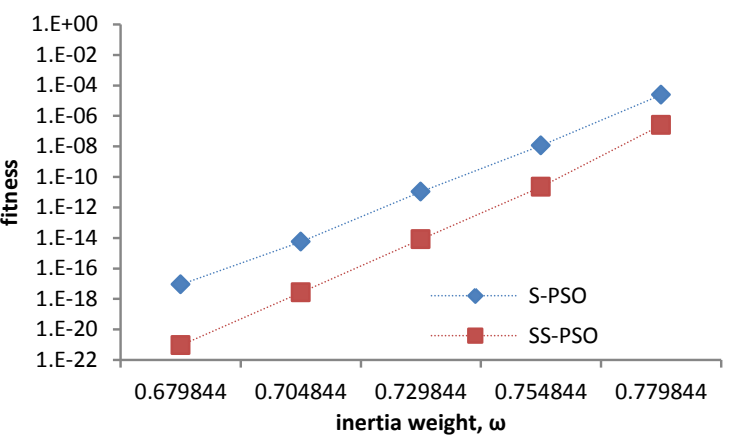

C

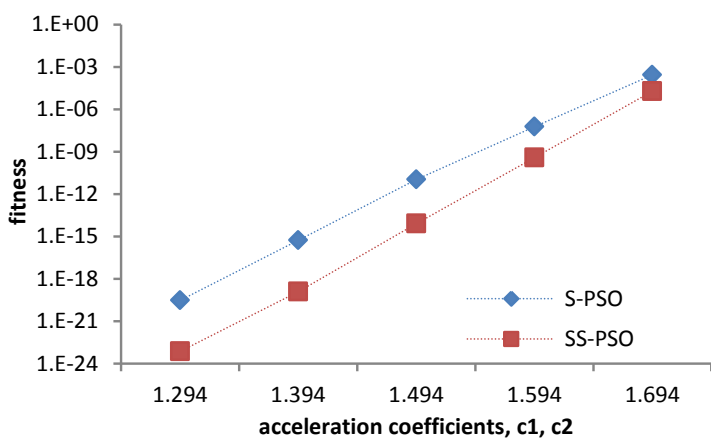

E

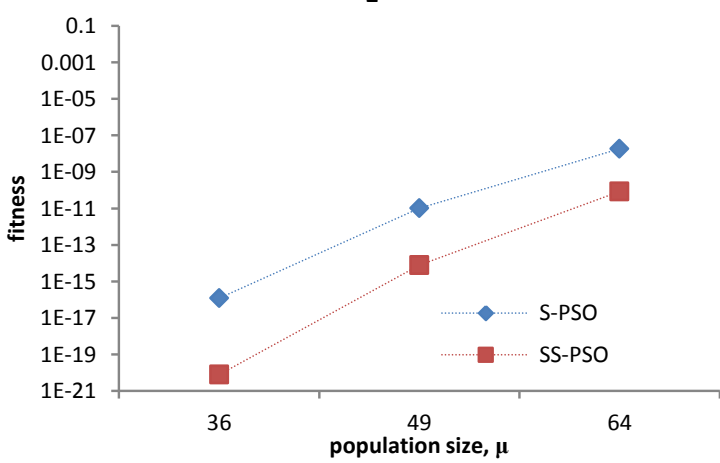

Manuscript to be reviewed

B
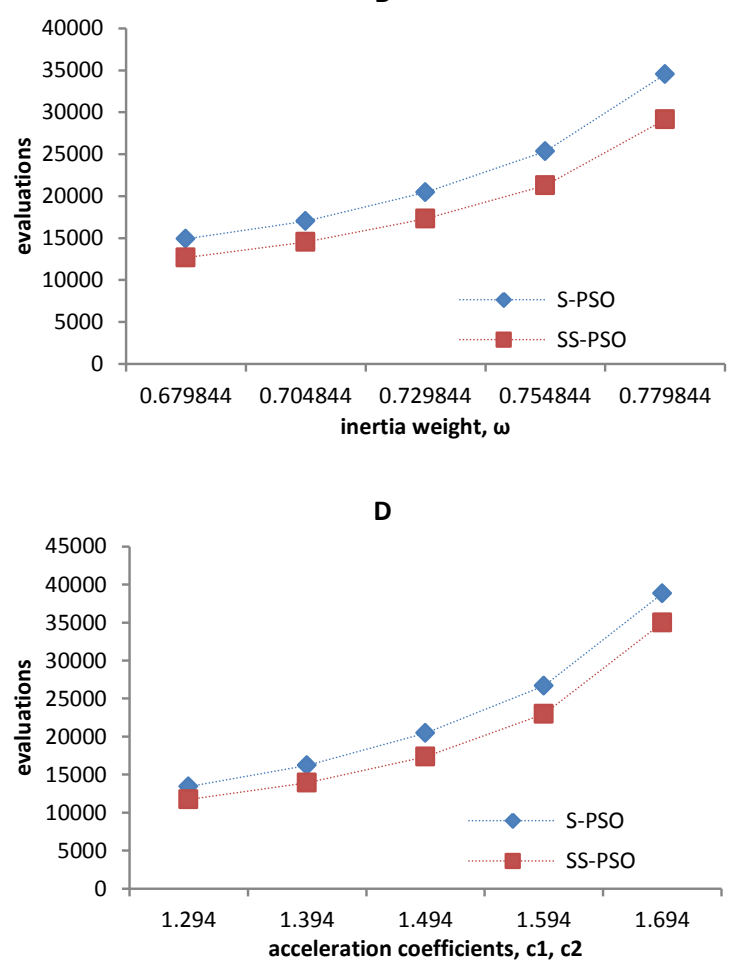

$\mathbf{F}$

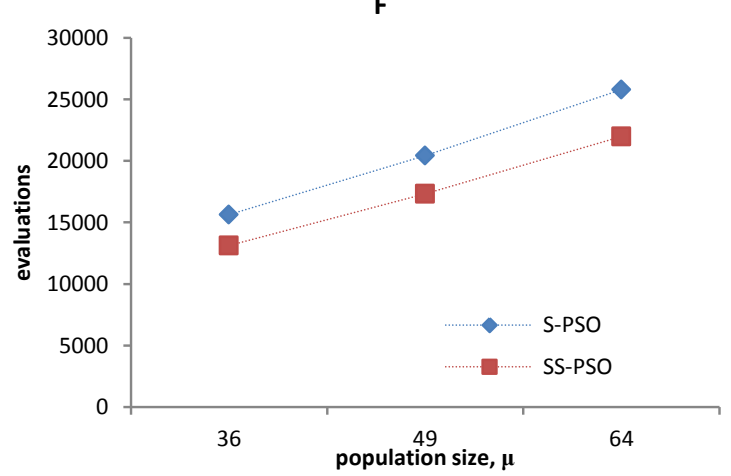


Figure $\mathbf{5}$ (on next page)

Parameters sensitivity: Ackley function (f8) 
PeerJ Computer Science

A
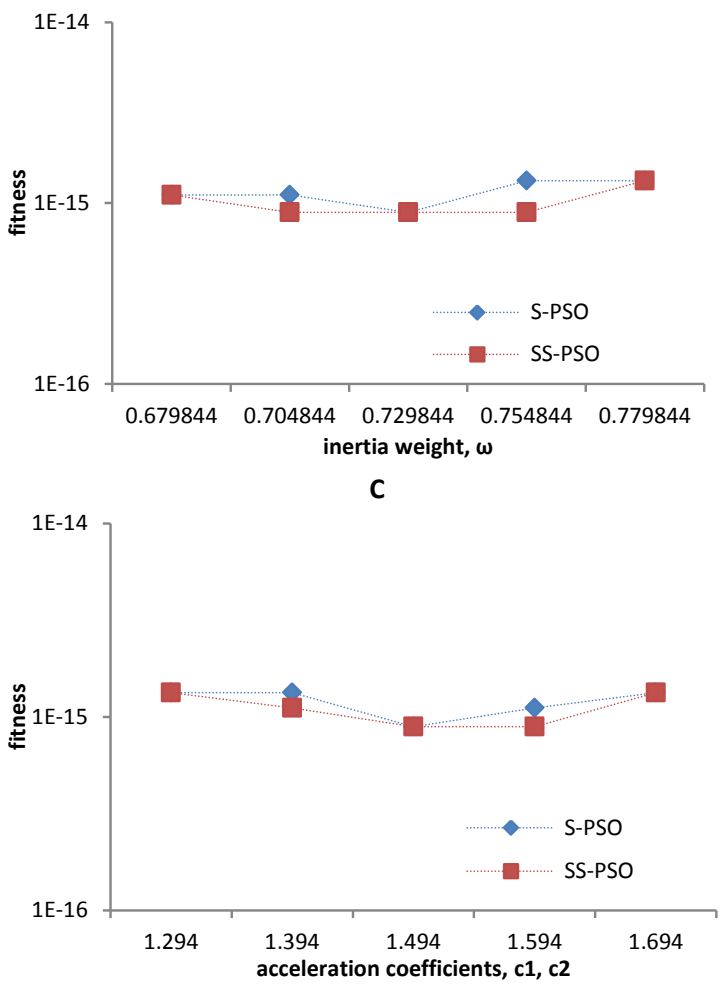

E

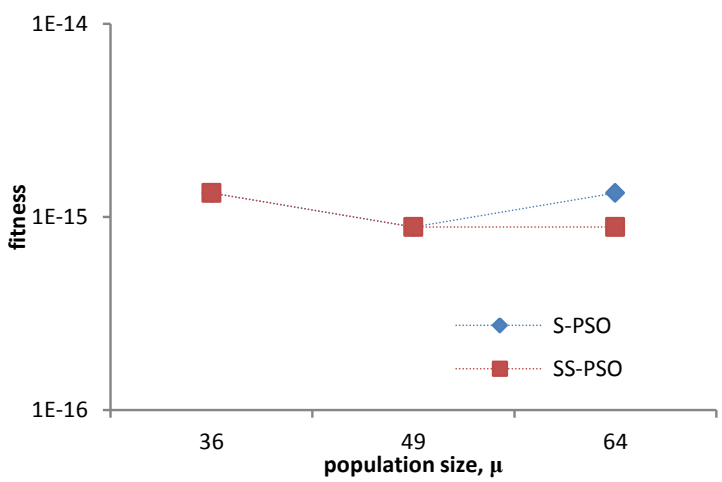

Manuscript to be reviewed

B

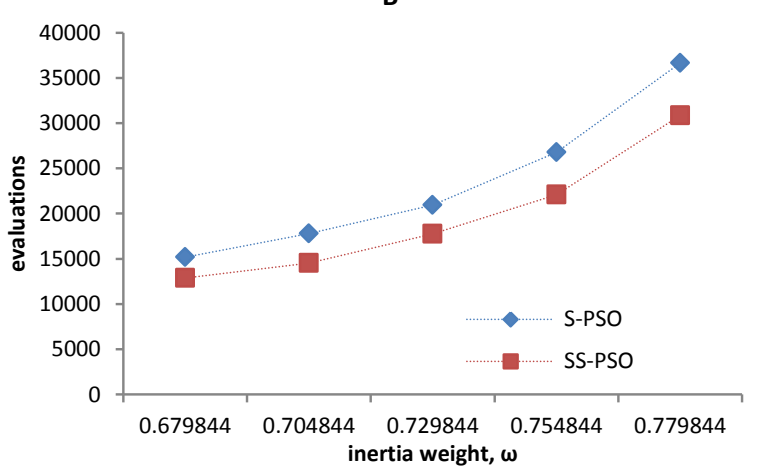

D

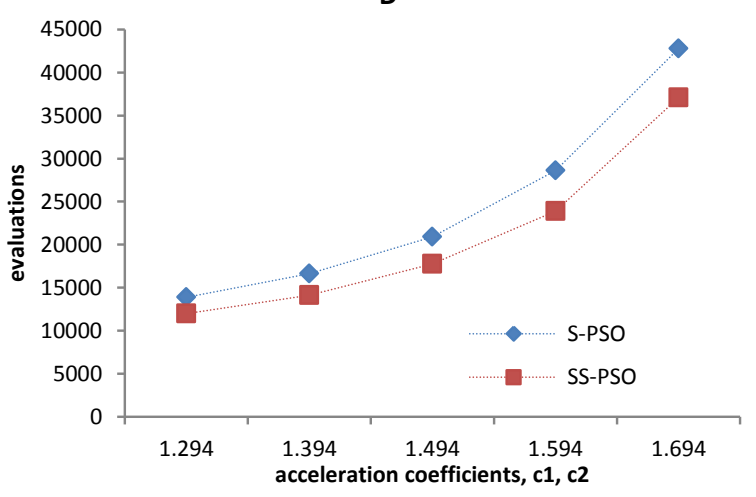

F

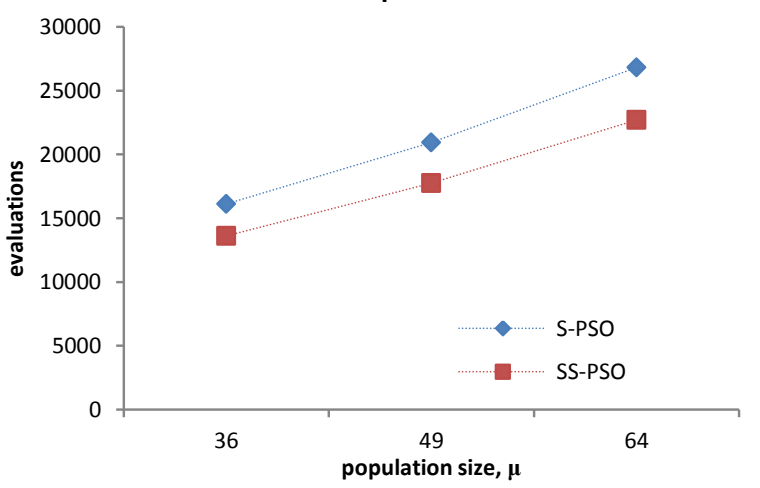


Figure 6 (on next page)

Parameters sensitivity: Shifted Quadric with noise. 
PeerJ Computer Science

A

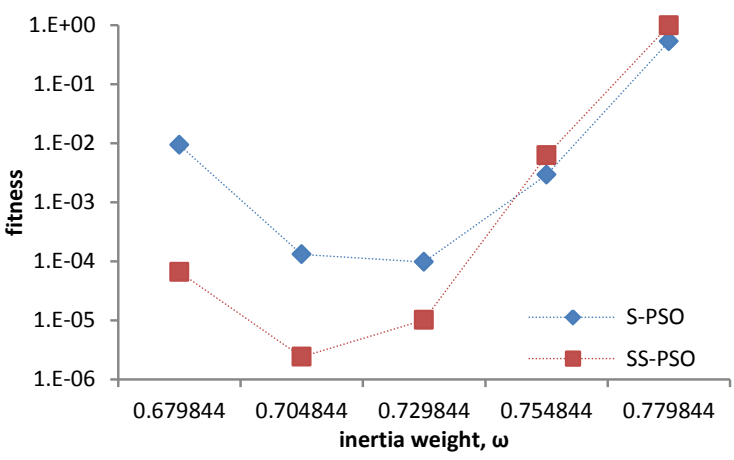

C

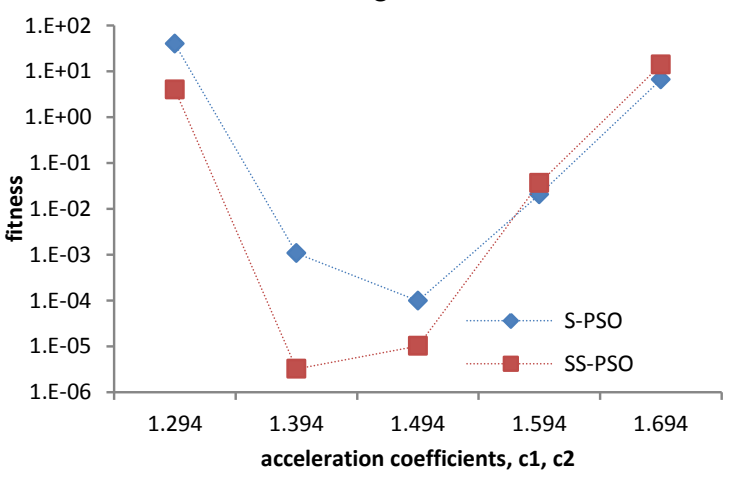

E

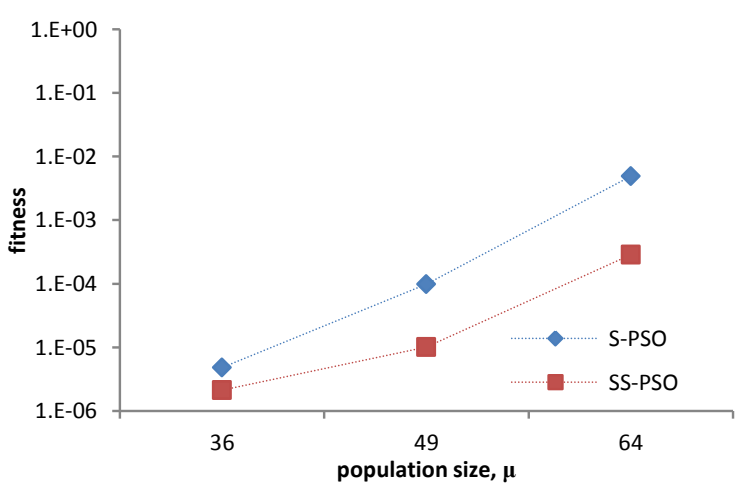

Manuscript to be reviewed

B

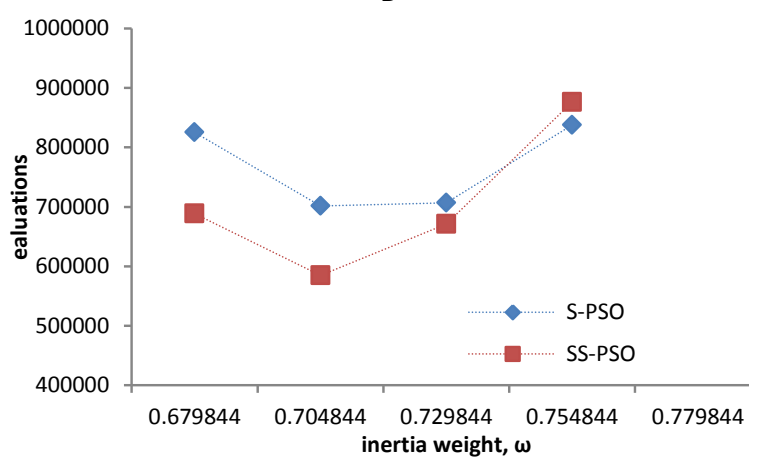

D

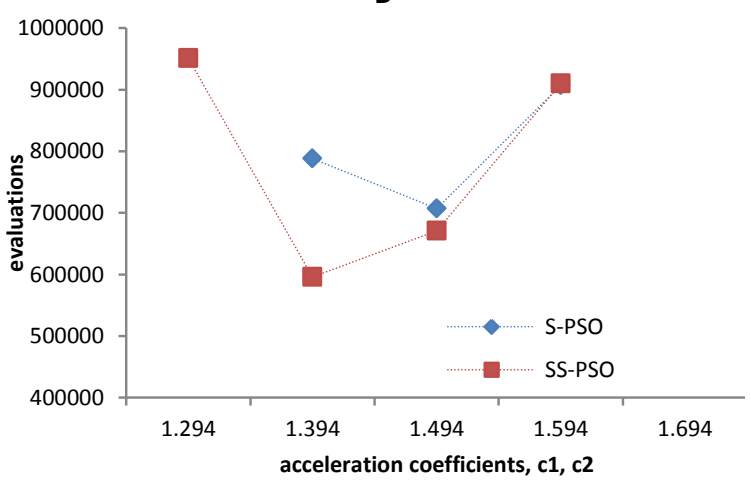

F

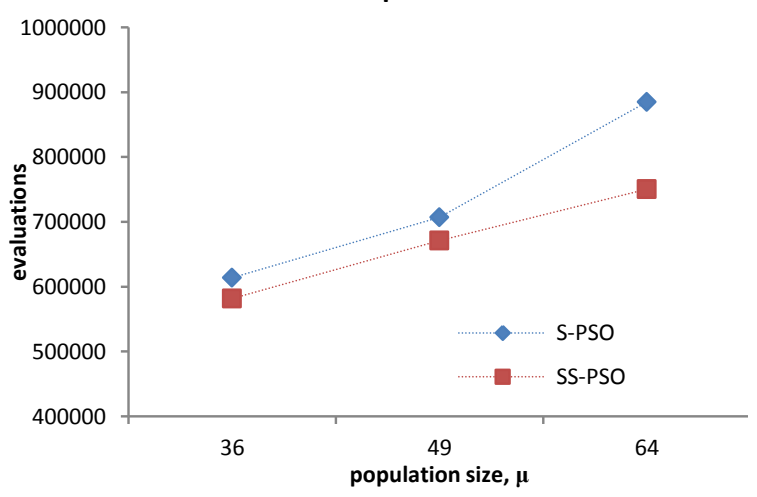


Figure 7 (on next page)

Parameters sensitivity: Rotated Griewank (f10). 
PeerJ Computer Science

A

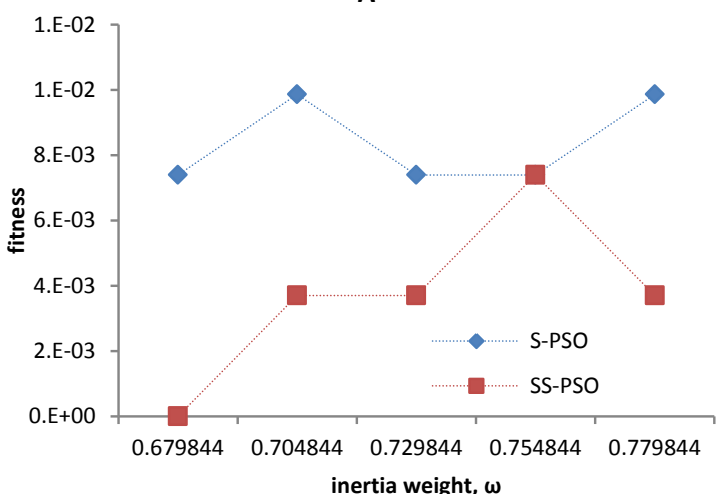

C

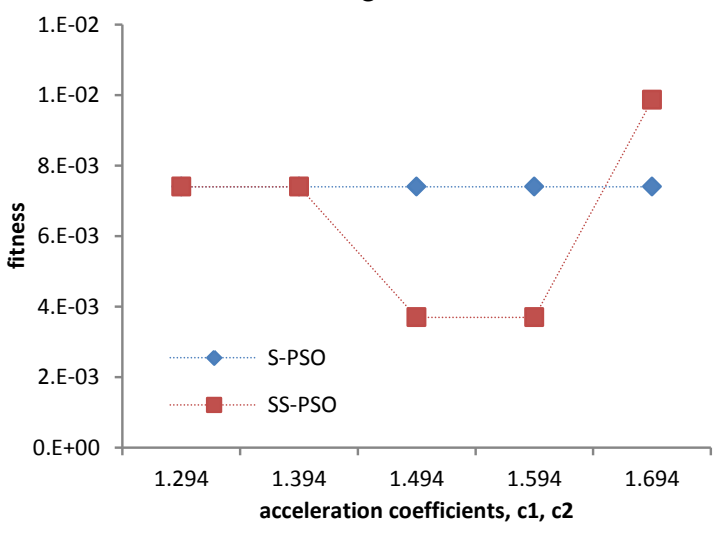

E

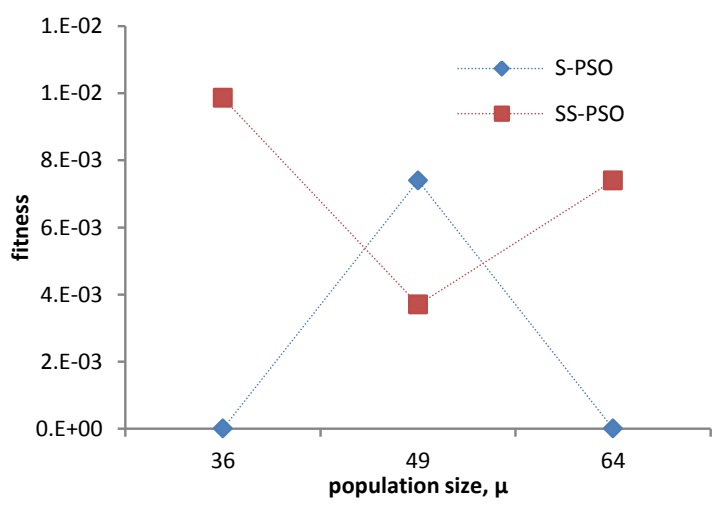

Manuscript to be reviewed

B

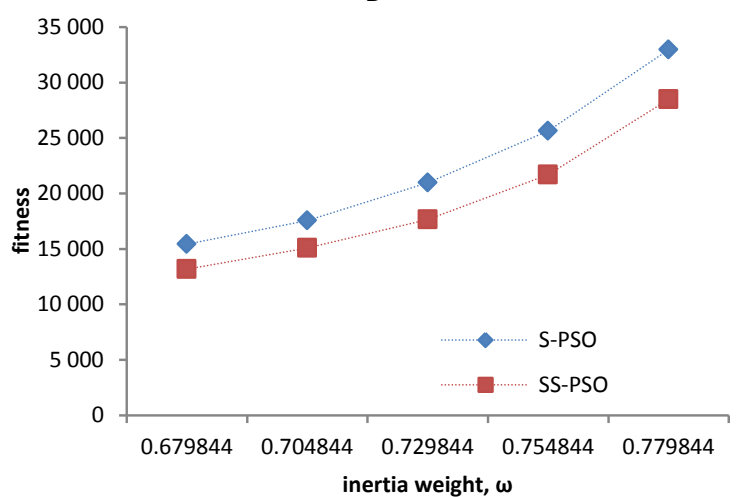

D

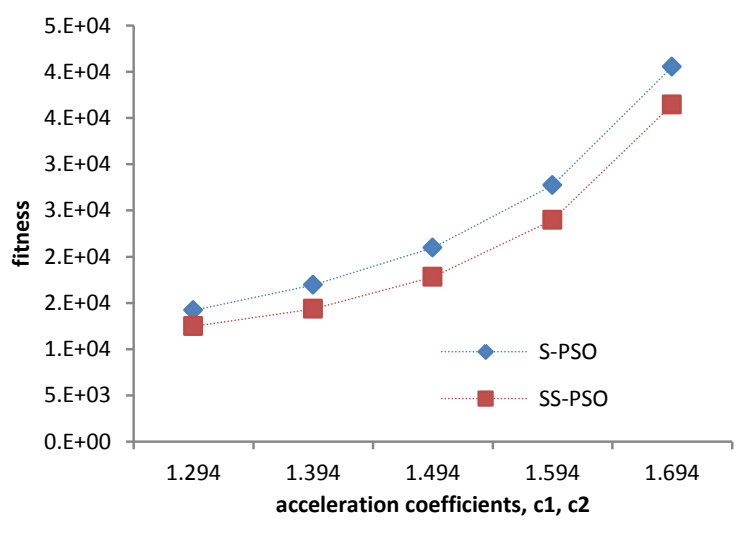

$\mathbf{F}$

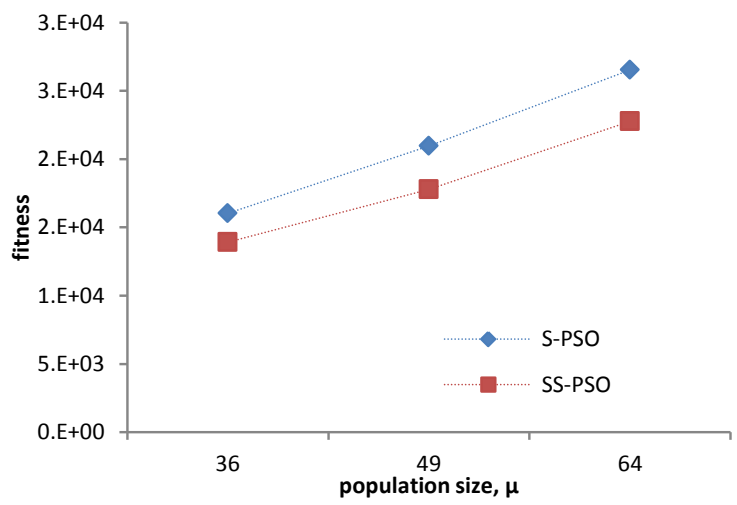


Figure 8 (on next page)

Regular graphs when population size is set to 8 . 
PeerJ Computer Science

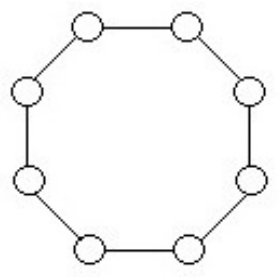

(A) $k=2$

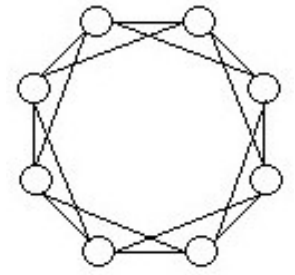

(B) $k=4$
Manuscript to be reviewed

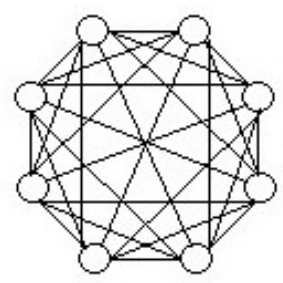

(C) $k=7=\mu-1$ 
Figure 9 (on next page)

SS-PSO with different topologies.

(A) Success rates. (B) mean fitness evaluations to a solution. 


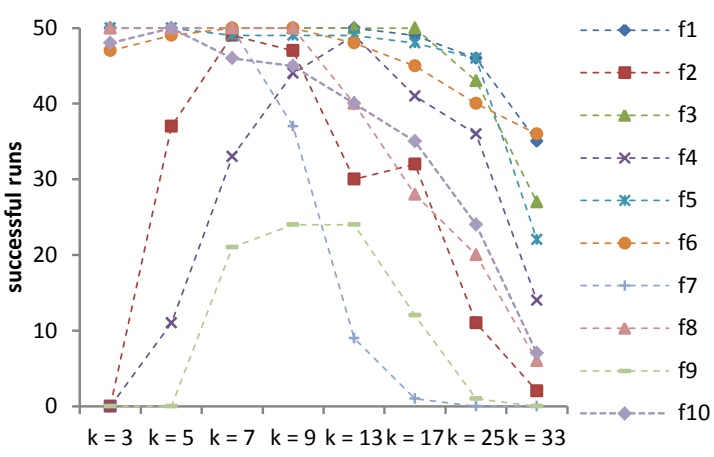

A

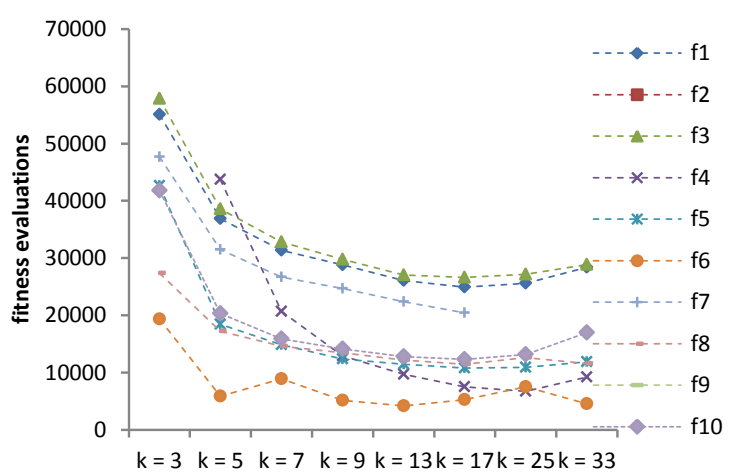

B 
Figure 10 (on next page)

S-PSO, SS-PSO and DSWPSO best fitness curves 
PeerJ Computer Science

(A) Sphere $\left(f_{1}\right)$

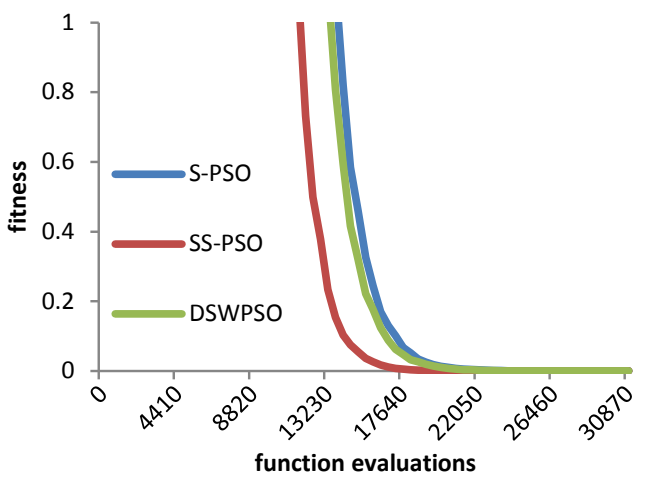

\section{Manuscript to be reviewed}

(B) Weierstrass $\left(f_{7}\right)$

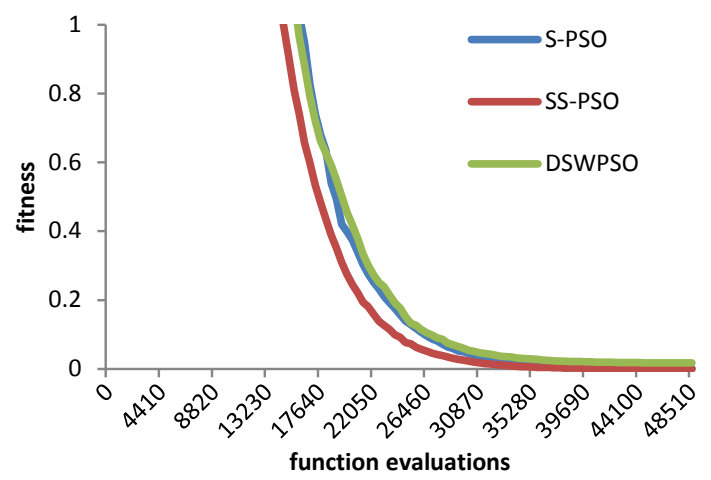


Figure 11(on next page)

S-PSO and SS-PSO running times 


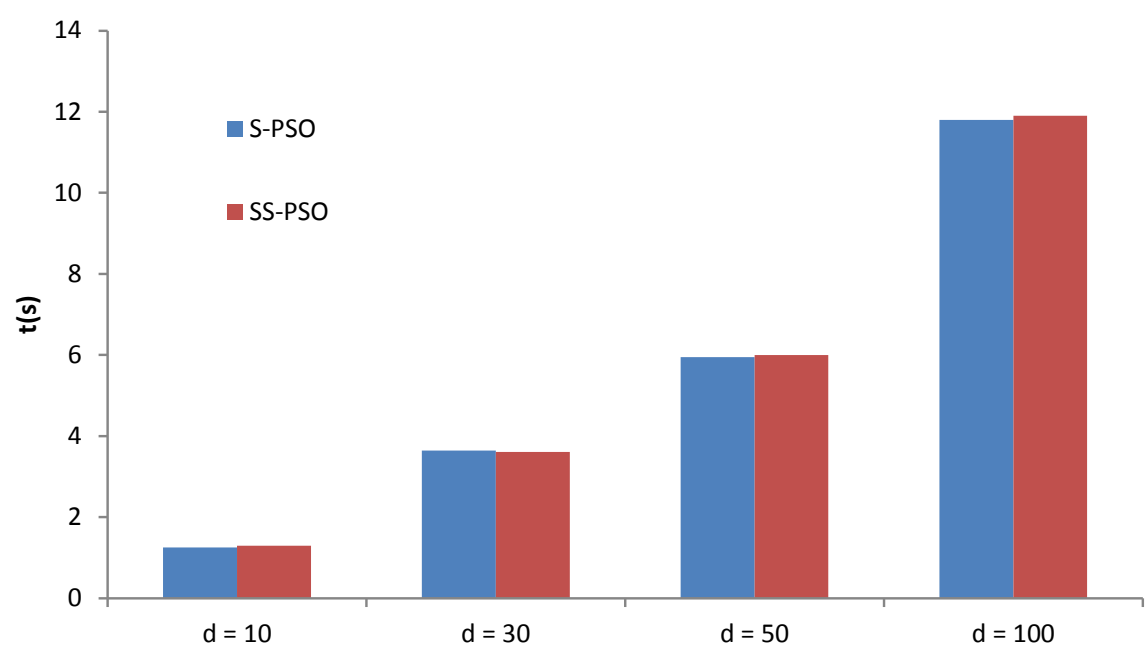




\section{Table $\mathbf{1}$ (on next page)}

Benchmark functions. 


\begin{tabular}{|c|c|c|c|}
\hline & mathematical representation & $\begin{array}{l}\text { Range of search/ } \\
\text { initialization }\end{array}$ & $\begin{array}{c}\text { stop } \\
\text { criterion }\end{array}$ \\
\hline $\begin{array}{c}\text { Sphere } \\
f_{l}\end{array}$ & $f_{1}(\vec{x})=\sum_{i=1}^{D} x_{i}^{2}$ & $\begin{array}{c}(-100,100)^{D} \\
(50,100)^{D}\end{array}$ & 0.01 \\
\hline $\begin{array}{l}\text { Quadric } \\
\qquad f_{2}\end{array}$ & $f_{2}(\vec{x})=\sum_{i=1}^{D}\left(\sum_{j=1}^{i} x_{j}\right)$ & $\begin{array}{c}(-100,100)^{D} \\
(50,100)^{D}\end{array}$ & 0.01 \\
\hline $\begin{array}{c}\text { Hyper } \\
\text { Ellipsoid } \\
f_{3}\end{array}$ & $f_{1}(\vec{x})=\sum_{i=1}^{D} i x_{i}^{2}$ & $\begin{array}{c}(-100,100)^{D} \\
(50,100)^{D}\end{array}$ & 0.01 \\
\hline $\begin{array}{c}\text { Rastrigin } \\
f_{4}\end{array}$ & $\left.2-10 \cos \left(2 \pi x_{i}\right)+10\right)$ & $\begin{array}{l}(-10,10)^{D} \\
(2.56,5.12)^{D}\end{array}$ & 100 \\
\hline $\begin{array}{c}\text { Griewank } \\
f_{5}\end{array}$ & $f_{5}(\vec{x})=1+\frac{1}{4000} \sum_{i=1}^{D} x_{i}^{2}-\prod_{i=1}^{D} \cos \left(\frac{x_{i}}{\sqrt{i}}\right)$ & $\begin{array}{c}(-600,600)^{D} \\
(300,600)^{D}\end{array}$ & 0.05 \\
\hline $\begin{array}{c}\text { Schaffer } \\
f_{6}\end{array}$ & $f_{6}(\vec{x})=0.5+\frac{\left(\sin \sqrt{x^{2}+y^{2}}\right)^{2}-0.5}{\left(1.0+0.001\left(x^{2}+y^{2}\right)\right)^{2}}$ & $\begin{array}{c}(-100,100)^{2} \\
(15,30)^{2}\end{array}$ & 0.00001 \\
\hline $\begin{array}{c}\text { Weierstrass } \\
\qquad f_{7}\end{array}$ & $\begin{array}{c}f_{7}(\vec{x})=\sum_{i=1}^{D}\left(\sum_{k=0}^{k \max }\left[a^{k} \cos \left(2 \pi b^{k}\left(x_{i}+0.5\right)\right)\right]\right)-D \sum_{k=0}^{k \max }\left[a^{k} \cos \left(2 \pi b^{k} \cdot 0.5\right)\right] \\
a=0.5, b=3, k \max =20\end{array}$ & $\begin{array}{l}(-0.5,0.5)^{D} \\
(-0.5,0.2)^{D}\end{array}$ & 0.01 \\
\hline $\begin{array}{c}\text { Ackley } \\
f_{8}\end{array}$ & $f_{8}(\vec{x})=-20 \exp \left(-0.2 \sqrt{\frac{1}{D} \sum_{i=1}^{D} x_{i}^{2}}\right)-\exp \left(\frac{1}{D} \sum_{i=1}^{D} \cos \left(2 \pi x_{i}\right)\right)+20+e$ & $\begin{array}{c}(-32.768,32.768)^{D} \\
(2.56,5.12)^{D}\end{array}$ & 0.01 \\
\hline $\begin{array}{l}\text { Shifted Quadric } \\
\text { with noise } \\
\qquad f_{9}\end{array}$ & $\begin{array}{c}f_{9}(\vec{z})=\sum_{i=1}^{D}\left(\sum_{j=1}^{i} z_{j}\right)^{2} *(1+0.4|N(0,1)|) \\
\vec{z}=\vec{x}-\vec{o}, \vec{o}=\left[o_{1}, \ldots o_{D}\right]: \text { shifted global optimum }\end{array}$ & $\begin{array}{c}(-100,100)^{D} \\
(50,100)^{D}\end{array}$ & 0.01 \\
\hline $\begin{array}{l}\text { Rotated } \\
\text { Griewank } \\
\qquad f_{10}\end{array}$ & $\begin{array}{c}f_{10}(\vec{z})=1+\frac{1}{4000} \sum_{i=1}^{D} z_{i}^{2}-\prod_{i=1}^{D} \cos \left(\frac{z_{i}}{\sqrt{i}}\right), \quad \vec{z}=\boldsymbol{M} \vec{x}, \mathbf{M} \text { :ortoghonal } \\
\text { matrix }\end{array}$ & $\begin{array}{l}(-600,600)^{D} \\
(300,600)^{D}\end{array}$ & 0.05 \\
\hline
\end{tabular}




\section{Table 2 (on next page)}

Median, minimum and maximum best fitness (50 runs). 


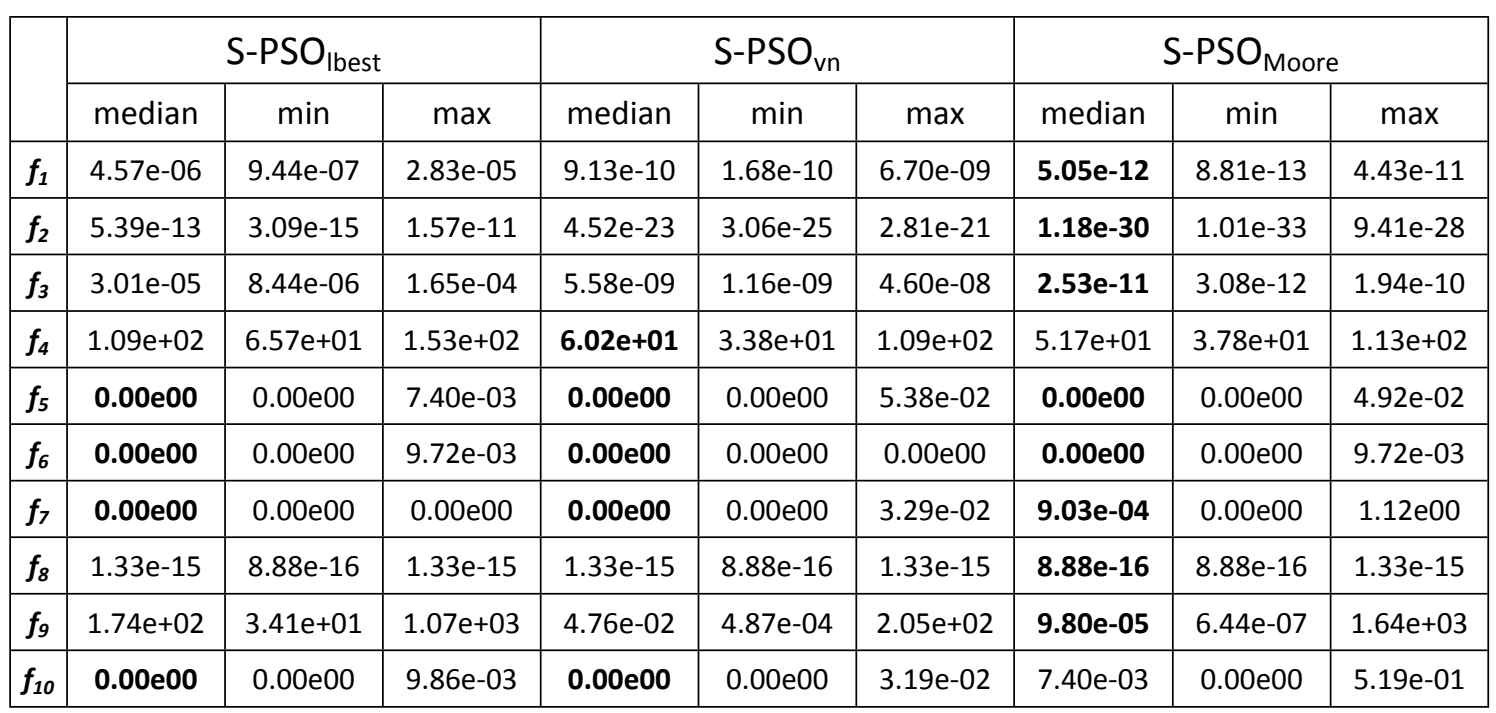




\section{Table 3 (on next page)}

Median, minimum and maximum evaluations required to meet the criteria (50 runs). 


\begin{tabular}{|c|c|c|c|c|c|c|c|c|c|}
\hline & \multicolumn{3}{|c|}{ S-PSO $_{\text {lbest }}$} & \multicolumn{3}{c|}{ S-PSO $_{\mathrm{vn}}$} & \multicolumn{3}{c|}{ S-PSO Moore } \\
\hline & median & $\min$ & $\max$ & median & $\min$ & $\max$ & median & $\min$ & $\max$ \\
\hline $\boldsymbol{f}_{\boldsymbol{1}}$ & 32511.5 & 30135 & 34937 & 23544.5 & 21952 & 24990 & $\mathbf{2 0 2 1 2}$ & 18669 & 22050 \\
\hline $\boldsymbol{f}_{\boldsymbol{2}}$ & 365270 & 313551 & 403858 & 217854 & 188111 & 242893 & $\mathbf{1 7 3 1 1 7}$ & 142688 & 194530 \\
\hline $\boldsymbol{f}_{\boldsymbol{3}}$ & 36799 & 34496 & 40425 & 26827 & 25029 & 29253 & $\mathbf{2 3 1 0 4}$ & 21462 & 24353 \\
\hline $\boldsymbol{f}_{\boldsymbol{4}}$ & 77518 & 21462 & 866173 & 15582 & 9604 & 74872 & $\mathbf{1 3 5 2 4 . 0}$ & 7448 & 49392 \\
\hline $\boldsymbol{f}_{5}$ & 31213 & 27244 & 34594 & 22736 & 20188 & 25333 & $\mathbf{1 9 3 7 9 . 5}$ & 17248 & 23765 \\
\hline $\boldsymbol{f}_{\boldsymbol{6}}$ & 18865 & 5243 & 145334 & 12323.5 & 3626 & 80213 & $\mathbf{7 1 0 5 . 0}$ & 3822 & 39788 \\
\hline $\boldsymbol{f}_{\boldsymbol{7}}$ & 62377 & 56399 & 69776 & 41356 & 37191 & 45766 & $\mathbf{3 3 4 9 2}$ & 31801 & 42973 \\
\hline $\boldsymbol{f}_{\boldsymbol{g}}$ & 35206.5 & 31556 & 39249 & 24206 & 22834 & 28928 & $\mathbf{2 0 9 2 3 . 0}$ & 19012 & 24794 \\
\hline $\boldsymbol{f}_{\boldsymbol{g}}$ & - & $\cdot$ &. & 883911 & 758961 & 976962 & $\mathbf{7 0 6 9 7 2}$ & 453201 & 922327 \\
\hline $\boldsymbol{f}_{10}$ & 33001.5 & 30331 & 37926 & 24157 & 21805 & 26460 & $\mathbf{2 1 0 2 1}$ & 18865 & 29939 \\
\hline
\end{tabular}




\section{Table 4(on next page)}

Success rates. 


\begin{tabular}{|c|c|c|c|}
\hline & S-PSO $_{\text {lbest }}$ & S-PSO $_{\mathrm{vn}}$ & S-PSO $_{\text {Moore }}$ \\
\hline$f_{1}$ & $\mathbf{5 0}$ & $\mathbf{5 0}$ & $\mathbf{5 0}$ \\
\hline$f_{2}$ & $\mathbf{5 0}$ & $\mathbf{5 0}$ & $\mathbf{5 0}$ \\
\hline$f_{3}$ & $\mathbf{5 0}$ & $\mathbf{5 0}$ & $\mathbf{5 0}$ \\
\hline$f_{4}$ & 17 & $\mathbf{4 9}$ & $\mathbf{4 9}$ \\
\hline$f_{5}$ & $\mathbf{5 0}$ & $\mathbf{5 0}$ & $\mathbf{5 0}$ \\
\hline$f_{6}$ & $\mathbf{5 0}$ & $\mathbf{5 0}$ & $\mathbf{5 0}$ \\
\hline$f_{7}$ & $\mathbf{5 0}$ & 47 & 34 \\
\hline$f_{8}$ & $\mathbf{5 0}$ & $\mathbf{5 0}$ & 50 \\
\hline$f_{9}$ & 6 & 9 & $\mathbf{4 7}$ \\
\hline$f_{10}$ & $\mathbf{5 0}$ & $\mathbf{5 0}$ & 47 \\
\hline
\end{tabular}

1 


\section{Table 5 (on next page)}

Fitness rank by Friedman test (with 0.05 signi $\square$ cance level).

The table gives the rank of each algorithm and in parenthesis the algorithms to which the di $\square$ erences are signi $\square$ cant according to the Friedman test. 


\begin{tabular}{|c|c|c|c|c|}
\hline & S-PSO $_{\text {Ibest }}(1)$ & S-PSO $_{\text {vn }}(2)$ & S-PSO $_{\text {Moore }}(3)$ & P value \\
\hline$f_{1}$ & $3.0(2)(3)$ & $2.0(1)(3)$ & $1.0(1)(2)$ & $<0.0001$ \\
\hline$f_{2}$ & $3.0(2)(3)$ & $2.0(1)(3)$ & $1.0(1)(2)$ & $<0.0001$ \\
\hline$f_{3}$ & $3.0(2)(3)$ & $2.0(1)(3)$ & $1.0(1)(2)$ & $<0.0001$ \\
\hline$f_{4}$ & $2.98(2)(3)$ & $1.47(1)$ & $1.55(1)$ & $<0.0001$ \\
\hline$f_{5}$ & $1.57(2)(3)$ & $2.03(1)(2)$ & $2.40(1)(3)$ & $<0.0001$ \\
\hline$f_{6}$ & $2.24(2)(3)$ & $1.94(1)$ & $1.82(1)$ & 0.00025 \\
\hline$f_{7}$ & $1.57(3)$ & $1.78(3)$ & $2.65(1)(3)$ & $<0.0001$ \\
\hline$f_{8}$ & $2.44(2)(3)$ & $1.96(1)(3)$ & $1.60(1)(2)$ & $<0.0001$ \\
\hline$f_{9}$ & $2.96(2)(3)$ & $1.98(1)(3)$ & $1.06(1)(2)$ & $<0.0001$ \\
\hline$f_{10}$ & $1.61(2)(3)$ & $1.99(2)(3)$ & $2.40(1)(2)$ & $<0.0001$ \\
\hline
\end{tabular}




\section{Table 6(on next page)}

Convergence speed rank by Friedman test (with 0.05 signi $\square$ cance level).

The table gives the rank of each algorithm and in parenthesis the algorithms to which the di $\square$ erences are signi $\square$ cant according to the Friedman test. 


\begin{tabular}{|c|c|c|c|c|}
\hline & S-PSO $_{\text {lbest }}(1)$ & S-PSO $_{\text {vn }}(2)$ & S-PSO $_{\text {Moore }}(3)$ & P value \\
\hline$f_{1}$ & $3.0(2)(3)$ & $1.99(1)(3)$ & $1.01(1)(2)$ & $<0.0001$ \\
\hline$f_{2}$ & $3.0(2)(3)$ & $1.98(1)(3)$ & $1.02(1)(2)$ & $<0.0001$ \\
\hline$f_{3}$ & $3.0(2)(3)$ & $1.99(1)(3)$ & $1.01(1)(2)$ & $<0.0001$ \\
\hline$f_{5}$ & $3.0(2)(3)$ & $1.96(1)(3)$ & $1.04(1)(2)$ & $<0.0001$ \\
\hline$f_{6}$ & $2.35(3)$ & $2.07(3)$ & $1.58(1)(2)$ & 0.00039 \\
\hline$f_{8}$ & $3.00(2)(3)$ & $2.00(1)(3)$ & $1.00(1)(2)$ & $<0.0001$ \\
\hline
\end{tabular}

1 


\section{Table 7 (on next page)}

SS-PSO ${ }_{\text {Moore }}$ results: solutions quality, convergence speed and success rates. 


\begin{tabular}{|c|c|c|c|c|c|c|c|}
\hline & \multicolumn{3}{|c|}{ fitness } & \multicolumn{3}{|c|}{ evaluations } \\
\hline & median & $\min$ & $\max$ & median & $\min$ & $\max$ & SR \\
\hline$f_{1}$ & $5.42 \mathrm{e}-15$ & $3.45 \mathrm{e}-16$ & $6.49 \mathrm{e}-14$ & 17019 & 15327 & 18819 & 50 \\
\hline$f_{2}$ & $7.18 \mathrm{e}-54$ & $8.41 \mathrm{e}-60$ & $4.87 \mathrm{e}-49$ & 133191 & 102258 & 163251 & 50 \\
\hline$f_{3}$ & $2.99 \mathrm{e}-14$ & $1.15 \mathrm{e}-15$ & $2.97 \mathrm{e}-13$ & 19768.5 & 17460 & 21069 & 50 \\
\hline$f_{4}$ & $5.12 \mathrm{e}+01$ & $2.19 \mathrm{e}+01$ & $1.04 \mathrm{e}+02$ & 14256 & 7659 & 58248 & 49 \\
\hline$f_{5}$ & $7.40 \mathrm{e}-03$ & $0.00 \mathrm{e} 00$ & $3.69 \mathrm{e}-02$ & 16884 & 14814 & 24291 & 50 \\
\hline$f_{6}$ & $0.00 \mathrm{e} 00$ & $0.00 \mathrm{e} 00$ & $0.00 \mathrm{e} 00$ & 6381 & 2727 & 21744 & 50 \\
\hline$f_{7}$ & $0.00 \mathrm{e} 00$ & $0.00 \mathrm{e} 00$ & $1.32 \mathrm{e}-01$ & 30717 & 28089 & 34254 & 48 \\
\hline$f_{8}$ & $8.88 \mathrm{e}-16$ & $8.88 \mathrm{e}-16$ & $1.33 \mathrm{e}-15$ & 17752.5 & 15750 & 19809 & 50 \\
\hline$f_{9}$ & $1.01 \mathrm{e}-05$ & $1.73 \mathrm{e}-08$ & $7.11 \mathrm{e}-04$ & 671175 & 425655 & 852786 & 50 \\
\hline$f_{10}$ & $3.70 \mathrm{e}-03$ & $0.00 \mathrm{e} 00$ & $5.24 \mathrm{e}-01$ & 17662.5 & 15669 & 27252 & 48 \\
\hline
\end{tabular}




\section{Table 8(on next page)}

Comparing S-PSO ${ }_{\text {Moore }}$ and SS-PSO ${ }_{\text {Moore }}$ with the Mann-Whitney tests.

+ if replace-worst ranks first in the Mann-Whitney test and the result is significant; - if replace-random ranks first and the results is significant; $\approx$ if the differences are not significant. 


\begin{tabular}{|c|c|c|c|c|c|c|c|c|c|c|}
\hline & $f_{1}$ & $f_{2}$ & $f_{3}$ & $f_{4}$ & $f_{5}$ & $f_{6}$ & $f_{7}$ & $f_{8}$ & $f_{9}$ & $f_{10}$ \\
\hline fitness & + & + & + & $\approx$ & $\approx$ & + & + & $\approx$ & + & $\approx$ \\
\hline evaluations & + & + & + & $\approx$ & + & $\approx$ & + & + & + & + \\
\hline
\end{tabular}

1 


\section{Table 9 (on next page)}

Results of SS-PSO variants: median, min, max and success rates (SR) 


\begin{tabular}{|c|c|c|c|c|c|c|c|c|c|c|c|c|c|c|}
\hline & \multicolumn{6}{|c|}{ SS-PSO $_{\text {Moore }}$ (replace-best) } & & \multicolumn{6}{|c|}{ SS-PSO Moore $_{\text {(replace-random) }}$} & \\
\hline & \multicolumn{3}{|c|}{ fitness } & \multicolumn{3}{|c|}{ evaluations } & \multirow[b]{2}{*}{ SR } & \multicolumn{3}{|c|}{ fitness } & \multicolumn{3}{|c|}{ evaluations } & \\
\hline & median & $\min$ & $\max$ & median & $\min$ & $\max$ & & median & $\min$ & $\max$ & median & $\min$ & $\max$ & $S R$ \\
\hline$f_{1}$ & $4.09 e-29$ & $2.50 \mathrm{e}-33$ & $2.00 e+04$ & 9468 & 6714 & 24669 & 45 & $6.04 e-14$ & $7.86 e-14$ & $6.59 e-12$ & 18972 & 16425 & 20781 & 50 \\
\hline$f_{2}$ & $1.50 \mathrm{e}+04$ & $4.12 \mathrm{e}-89$ & $3.50 e+04$ & 66307 & 64251 & 68364 & 2 & $8.33 e-32$ & $4.59 e-34$ & $5.00 e+03$ & 170091 & 136062 & 195498 & 47 \\
\hline$f_{3}$ & $3.01 \mathrm{e}-27$ & $9.54 e-34$ & $1.00 \mathrm{e}+05$ & 11718 & 8208 & 36000 & 35 & $1.66 \mathrm{e}-12$ & $1.30 \mathrm{e}-13$ & $2.25 \mathrm{e}-11$ & 21118 & 19548 & 23283 & 50 \\
\hline$f_{4}$ & $1.30 \mathrm{e}+02$ & $7.46 e+01$ & $2.00 e+02$ & 15192 & 8964 & 108495 & 9 & $5.62 e+01$ & $2.39 e+01$ & $8.76 e+01$ & 11052 & 5679 & 23571 & 50 \\
\hline$f_{5}$ & $3.08 \mathrm{e}-02$ & $0.00 \mathrm{e} 00$ & $1.81 e+02$ & 10287 & 8694 & 26838 & 12 & $0.00 \mathrm{e} 00$ & $0.00 \mathrm{e} 00$ & $8.33 e-02$ & 19849.5 & 17748 & 26739 & 36 \\
\hline$f_{6}$ & $3.59 e-04$ & $0.00 \mathrm{e} 00$ & $9.72 \mathrm{e}-03$ & 39811.5 & 1242 & 140247 & 38 & $0.00 \mathrm{e} 00$ & $0.00 \mathrm{e} 00$ & $9.72 \mathrm{e}-03$ & 8460 & 3276 & 62091 & 50 \\
\hline$f_{7}$ & $7.52 \mathrm{e} 00$ & $2.64 \mathrm{e} 00$ & $1.57 e+01$ & - & - & - & 0 & $1.58 \mathrm{e}-03$ & $0.00 \mathrm{e} 00$ & $2.48 \mathrm{e} 00$ & 33912 & 31239 & 41211 & 30 \\
\hline$f_{8}$ & $2.28 \mathrm{e} 00$ & $8.86 e-16$ & $3.84 \mathrm{e} 00$ & 20898 & 13158 & 28764 & 6 & $1.11 \mathrm{e}-15$ & $8,86 e-16$ & $1.33 e-15$ & 19822.5 & 18252 & 25416 & 50 \\
\hline$f_{g}$ & $1.06 \mathrm{e}-01$ & $1.98 \mathrm{e}-03$ & $1.53 e+04$ & 902407 & 812736 & 949590 & 12 & $1.64 \mathrm{e}-04$ & $1.44 \mathrm{e}-06$ & $6.01 \mathrm{e}+01$ & 736713 & 546858 & 891432 & 49 \\
\hline$f_{10}$ & $1.04 \mathrm{e}+01$ & $0.00 \mathrm{e} 00$ & $4.04 e+02$ & 16065 & 8388 & 23742 & 2 & $3.70 \mathrm{e}-03$ & $0.00 \mathrm{e} 00$ & $5.09 e-01$ & 21915 & 18567 & 50607 & 39 \\
\hline
\end{tabular}




\section{Table $\mathbf{1 0}$ (on next page)}

Comparing replace-worst and replace-random with Mann-Whitney tests.

+ if replace-worst ranks first in the Mann-Whitney test and the result is significant; - if replace-random ranks first and the result is significant; $\approx$ if the differences are not significant. 


\begin{tabular}{|c|c|c|c|c|c|c|c|c|c|c|}
\hline & $f_{1}$ & $f_{2}$ & $f_{3}$ & $f_{4}$ & $f_{5}$ & $f_{6}$ & $f_{7}$ & $f_{8}$ & $f_{9}$ & $f_{10}$ \\
\hline fitness & + & + & + & $\approx$ & $\approx$ & $\approx$ & + & + & + & $\approx$ \\
\hline evaluations & + & + & + & - & + & + & + & + & + & + \\
\hline
\end{tabular}

1 


\section{Table 11 (on next page)}

Solutions quality with different problem dimension. 


\begin{tabular}{|c|c|c|c|c|c|c|c|c|c|c|}
\hline & \multicolumn{2}{|c|}{$D=10$} & \multicolumn{2}{|c|}{$D=20$} & \multicolumn{2}{|c|}{$D=30$} & \multicolumn{2}{|c|}{$D=40$} & \multicolumn{2}{|c|}{$D=50$} \\
\hline & S-PSO & SS-PSO & S-PSO & SS-PSO & S-PSO & SS-PSO & S-PSO & SS-PSO & S-PSO & SS-PSO \\
\hline$f_{1}$ & $1.06 e-37$ & $2.71 e-47$ & $1.87 e-19$ & $5.72 e-24$ & $1.04 \mathrm{e}-11$ & $7.83 e-15$ & $7.15 e-08$ & $2.96 \mathrm{e}-10$ & $3.69 e-05$ & $2.01 \mathrm{e}-10$ \\
\hline$f_{2}$ & $0.00 \mathrm{e} 00$ & $0.00 \mathrm{e} 00$ & $4.63 e-82$ & $1.37 e-89$ & $1.17 e-30$ & $9.52 e-54$ & $1.18 e-13$ & $1.10 \mathrm{e}-20$ & $1.36 e-06$ & $2.36 \mathrm{e}-06$ \\
\hline$f_{3}$ & $1.57 e-40$ & $0.00 \mathrm{e} 00$ & $2.08 \mathrm{e}-19$ & $3.37 e-24$ & $2.76 e-11$ & $1.58 \mathrm{e}-14$ & 8.77e-07 & $2.58 \mathrm{e}-09$ & 4.59e-04 & $3.19 \mathrm{e}-06$ \\
\hline$f_{4}$ & $1.99 \mathrm{e} 00$ & $1.99 \mathrm{e} 00$ & $2.09 e+01$ & $2.04 e+01$ & $6.17 e+01$ & $5.12 \mathrm{e}+01$ & $1.01 \mathrm{e}+02$ & $1.06 e+02$ & $1.70 \mathrm{e}+02$ & $1.37 e+02$ \\
\hline$f_{5}$ & $2.83 e-02$ & $3.60 \mathrm{e}-02$ & $8.63 e-03$ & $1.11 \mathrm{e}-02$ & $0.00 \mathrm{e} 00$ & $7.40 \mathrm{e}-03$ & $0.00 \mathrm{e} 00$ & $7.40 \mathrm{e}-03$ & $0.00 \mathrm{e} 00$ & $0.00 \mathrm{e} 00$ \\
\hline$f_{7}$ & $0.00 \mathrm{e} 00$ & $0.00 \mathrm{e} 00$ & $0.00 \mathrm{e} 00$ & $0.00 \mathrm{e} 00$ & $9.03 e-04$ & $0.00 \mathrm{e} 00$ & $9.03 e-04$ & 3.39e-04 & $1.34 \mathrm{e}-01$ & $2.15 e-02$ \\
\hline$f_{8}$ & $4.44 \mathrm{e}-16$ & $4.44 e-16$ & $8.88 \mathrm{e}-16$ & $8.88 e-16$ & $8.88 e-16$ & $8.88 e-16$ & $1.33 e-15$ & $1.33 e-15$ & $1.33 e-15$ & $1.33 e-15$ \\
\hline$f_{g}$ & $0.00 \mathrm{e} 00$ & $0.00 \mathrm{e} 00$ & $1.92 \mathrm{e}-10$ & $1.32 \mathrm{e}-10$ & $9.8 e-05$ & $1.01 \mathrm{e}-05$ & $6.18 \mathrm{e}+01$ & $3.40 e+01$ & $1.34 \mathrm{e}+03$ & $1.70 e+03$ \\
\hline$f_{10}$ & $3.20 \mathrm{e}-02$ & $3.20 \mathrm{e}-02$ & - & - & $7.40 \mathrm{e}-03$ & $7.40 \mathrm{e}-03$ & - & - & $0.00 \mathrm{e} 00$ & $0.00 \mathrm{e} 00$ \\
\hline
\end{tabular}




\section{Table 12 (on next page)}

Success rates with different problem dimension. 


\begin{tabular}{|c|c|c|c|c|c|c|c|c|c|c|}
\hline & \multicolumn{2}{|c|}{$\mathrm{D}=10$} & \multicolumn{2}{c|}{$\mathrm{D}=20$} & \multicolumn{2}{c|}{$\mathrm{D}=30$} & \multicolumn{2}{c|}{$\mathrm{D}=40$} & \multicolumn{2}{c|}{$\mathrm{D}=50$} \\
\hline & S-PSO & SS-PSO & S-PSO & SS-PSO & S-PSO & SS-PSO & S-PSO & SS-PSO & S-PSO & SS-PSO \\
\hline$f_{1}$ & $\mathbf{5 0}$ & $\mathbf{5 0}$ & $\mathbf{5 0}$ & $\mathbf{5 0}$ & $\mathbf{5 0}$ & $\mathbf{5 0}$ & $\mathbf{5 0}$ & $\mathbf{5 0}$ & $\mathbf{5 0}$ & $\mathbf{5 0}$ \\
\hline$f_{2}$ & $\mathbf{5 0}$ & $\mathbf{5 0}$ & $\mathbf{5 0}$ & $\mathbf{5 0}$ & $\mathbf{5 0}$ & $\mathbf{5 0}$ & 43 & $\mathbf{5 0}$ & 32 & $\mathbf{4 8}$ \\
\hline$f_{3}$ & $\mathbf{5 0}$ & $\mathbf{5 0}$ & $\mathbf{5 0}$ & $\mathbf{5 0}$ & $\mathbf{5 0}$ & $\mathbf{5 0}$ & $\mathbf{5 0}$ & $\mathbf{5 0}$ & 50 & $\mathbf{5 0}$ \\
\hline$f_{4}$ & $\mathbf{5 0}$ & $\mathbf{5 0}$ & $\mathbf{5 0}$ & $\mathbf{5 0}$ & $\mathbf{4 9}$ & $\mathbf{4 9}$ & $\mathbf{2 5}$ & 21 & 0 & $\mathbf{3}$ \\
\hline$f_{5}$ & $\mathbf{4 0}$ & 37 & 49 & $\mathbf{5 0}$ & $\mathbf{5 0}$ & 47 & $\mathbf{5 0}$ & 49 & $\mathbf{5 0}$ & $\mathbf{5 0}$ \\
\hline$f_{7}$ & $\mathbf{5 0}$ & $\mathbf{5 0}$ & $\mathbf{4 9}$ & $\mathbf{4 9}$ & 34 & $\mathbf{4 8}$ & 8 & $\mathbf{3 5}$ & 4 & $\mathbf{1 9}$ \\
\hline$f_{8}$ & $\mathbf{5 0}$ & $\mathbf{5 0}$ & $\mathbf{5 0}$ & $\mathbf{5 0}$ & $\mathbf{5 0}$ & $\mathbf{5 0}$ & $\mathbf{5 0}$ & $\mathbf{5 0}$ & 46 & $\mathbf{5 0}$ \\
\hline$f_{9}$ & $\mathbf{5 0}$ & $\mathbf{5 0}$ & $\mathbf{5 0}$ & $\mathbf{5 0}$ & 47 & $\mathbf{5 0}$ & $\mathbf{0}$ & $\mathbf{0}$ & $\mathbf{0}$ & $\mathbf{0}$ \\
\hline$f_{10}$ & $\mathbf{4 4}$ & 35 & - & - & 46 & $\mathbf{4 8}$ & - & - & 48 & $\mathbf{4 9}$ \\
\hline
\end{tabular}




\section{Table $\mathbf{1 3}$ (on next page)}

PSO-TVAC results. 


\begin{tabular}{|c|c|c|c|c|c|c|c|}
\hline & \multicolumn{3}{|c|}{ fitness } & \multicolumn{3}{|c|}{ evaluations } & SR \\
\hline & median & $\min$ & $\max$ & $\operatorname{median}$ & $\min$ & $\max$ & \\
\hline$f_{1}$ & $2.85 \mathrm{e}-21$ & $2.55 \mathrm{e}-22$ & $1.84 \mathrm{e}-20$ & 11956 & 11221 & 13181 & 50 \\
\hline$f_{2}$ & $4.47 \mathrm{e}-51$ & $1.23 \mathrm{e}-54$ & $5.00 \mathrm{e} 03$ & 208740 & 185514 & 238532 & 49 \\
\hline$f_{\boldsymbol{3}}$ & $3.87 \mathrm{e}-21$ & $3.01 \mathrm{e}-22$ & $1.57 \mathrm{e}-19$ & 13769 & 12740 & 16121 & 50 \\
\hline$f_{4}$ & $3.08 \mathrm{e}+01$ & $1.11 \mathrm{e}+01$ & $5.8 \mathrm{e}+01$ & $\mathbf{3 1 1 1 4}$ & 16661 & 59388 & 50 \\
\hline$f_{5}$ & $\mathbf{0 . 0 0 e 0 0}$ & $0.00 \mathrm{e} 00$ & $4.91 \mathrm{e}-02$ & 15141 & 12642 & 91238 & 50 \\
\hline$f_{\boldsymbol{6}}$ & $\mathbf{0 . 0 0 e 0 0}$ & $0.00 \mathrm{e} 00$ & $0.00 \mathrm{e} 00$ & 11956 & 5145 & 38612 & 49 \\
\hline$f_{7}$ & $\mathbf{0 . 0 0 e 0 0}$ & $0.00 \mathrm{e} 00$ & $1.64 \mathrm{e}-01$ & 35280 & 31017 & 42336 & 49 \\
\hline$f_{\boldsymbol{g}}$ & $\mathbf{7 . 5 5 e}-1 \mathbf{1 5}$ & $4.00 \mathrm{e}-15$ & $7.55 \mathrm{e}-15$ & 21070 & 17346 & 29988 & 50 \\
\hline$f_{\boldsymbol{g}}$ & $6.14 \mathrm{e}-09$ & $1.74 \mathrm{e}-09$ & $6.28 \mathrm{e}-06$ & $\mathbf{2 2 7 0 6 6}$ & 199528 & 287042 & 47 \\
\hline$f_{10}$ & $7.40 \mathrm{e}-03$ & $0.00 \mathrm{e} 00$ & $5.24 \mathrm{e}-01$ & 18620 & 14602 & 87220 & 42 \\
\hline
\end{tabular}


Table 14(on next page)

SS-PSO-TVAC results. 


\begin{tabular}{|c|c|c|c|c|c|c|c|}
\hline & \multicolumn{3}{|c|}{ fitness } & \multicolumn{3}{|c|}{ evaluations } & \multirow{2}{*}{$S R$} \\
\hline & median & $\min$ & $\max$ & median & $\min$ & $\max$ & \\
\hline$f_{1}$ & $7.85 e-26$ & $4.82 \mathrm{e}-27$ & $2.35 e-24$ & 10417 & 9126 & 11322 & 50 \\
\hline$f_{2}$ & $5.18 e-63$ & $2.30 e-67$ & $5.77 e-60$ & 190458 & 168282 & 226062 & 50 \\
\hline$f_{3}$ & $1.66 \mathrm{e}-25$ & $7.76 e-27$ & $9.14 \mathrm{e}-24$ & 11925 & 10422 & 13923 & 50 \\
\hline$f_{4}$ & $3.48 e+01$ & $1.89 \mathrm{e} 01$ & $7.46 e+01$ & 38043 & 22032 & 108927 & 50 \\
\hline$f_{5}$ & $0.00 \mathrm{e} 00$ & $0.00 \mathrm{e} 00$ & $4.42 e-02$ & 13662 & 9963 & 56421 & 49 \\
\hline$f_{6}$ & $0.00 \mathrm{e} 00$ & $0.00 \mathrm{e} 00$ & $0.00 \mathrm{e} 00$ & 8421 & 2547 & 26325 & 49 \\
\hline$f_{7}$ & $0.00 \mathrm{e} 00$ & $0.00 \mathrm{e} 00$ & $2.62 \mathrm{e}-01$ & 31752 & 28323 & 41193 & 43 \\
\hline$f_{8}$ & $7.55 e-15$ & $4.00 e-15$ & $7.55 e-15$ & 18756 & 14958 & 23904 & 49 \\
\hline$f_{9}$ & 5.41e-09 & $6.37 e-10$ & $5.80 \mathrm{e}-03$ & 315792 & 192906 & 476532 & 48 \\
\hline$f_{10}$ & $0.00 \mathrm{e} 00$ & $0.00 \mathrm{e} 00$ & $3.93 e-02$ & 15948 & 12762 & 75510 & 40 \\
\hline
\end{tabular}




\section{Table $\mathbf{1 5}$ (on next page)}

Comparing SS-PSO-TVAC and PSO-TVAC with Mann-Whitney tests.

+ if SS-PSO-TVAC ranks first in the Mann-Whitney test and the result is significant; - if PSO-

TVAC ranks first and the results is significant; $\approx$ if the differences are not significant. 


\begin{tabular}{|c|c|c|c|c|c|c|c|c|c|c|}
\hline & $f_{1}$ & $f_{2}$ & $f_{3}$ & $f_{4}$ & $f_{5}$ & $f_{6}$ & $f_{7}$ & $f_{8}$ & $f_{9}$ & $f_{10}$ \\
\hline fitness & + & + & + & $\approx$ & $\approx$ & $\approx$ & $\approx$ & $\approx$ & $\approx$ & $\approx$ \\
\hline evaluations & + & + & + & $\approx$ & $\approx$ & + & + & + & - & + \\
\hline
\end{tabular}




\section{Table $\mathbf{1 6}$ (on next page)}

CLPSO results. 


\begin{tabular}{|c|c|c|c|c|c|c|c|}
\hline & \multicolumn{3}{|c|}{ fitness } & \multicolumn{4}{|c|}{ evaluations } \\
\hline & median & $\min$ & $\max$ & median & $\min$ & $\max$ & SR \\
\hline$f_{1}$ & $9.59 e-07$ & $4.00 e-07$ & $2.23 e-06$ & 34848 & 33355 & 35909 & 50 \\
\hline$f_{2}$ & $2.16 e-01$ & $8.66 e-02$ & $5.46 e-01$ & - & - & - & - \\
\hline$f_{3}$ & $2.31 e-06$ & $1.18 \mathrm{e}-06$ & $4.96 e-06$ & 36777 & 35665 & 37972 & 50 \\
\hline$f_{4}$ & $4.97 \mathrm{e} 00$ & $1.99 \mathrm{e} 00$ & $1.20 \mathrm{e}+01$ & 115701 & 94674 & 129493 & 50 \\
\hline$f_{5}$ & $0.00 \mathrm{e} 00$ & $0.00 \mathrm{e} 00$ & $9.74 \mathrm{e}-13$ & 199537 & 164774 & 243806 & 50 \\
\hline$f_{6}$ & $6.49 e-06$ & 7.69e-09 & $1.13 e-04$ & 81149 & 37710 & 96320 & 31 \\
\hline$f_{7}$ & $8.67 e-13$ & $3.48 \mathrm{e}-13$ & $1.61 e-12$ & 430069 & 418700 & 440035 & 50 \\
\hline$f_{8}$ & $7.55 e-15$ & $4.00 \mathrm{e}-15$ & $7.55 e-15$ & 282613 & 275897 & 285290 & 50 \\
\hline$f_{9}$ & $4.36 e-01$ & $1.51 \mathrm{e}-01$ & $1.11 \mathrm{e} 00$ & - & - & - & - \\
\hline$f_{10}$ & $0.00 \mathrm{e} 00$ & $0.00 \mathrm{e} 00$ & $2.26 \mathrm{e}-14$ & 173346 & 151269 & 229975 & 50 \\
\hline
\end{tabular}


Table $\mathbf{1 7}$ (on next page)

SS-CLPSO results. 


\begin{tabular}{|c|c|c|c|c|c|c|c|}
\hline & \multicolumn{3}{|c|}{ fitness } & \multicolumn{4}{|c|}{ evaluations } \\
\hline & median & $\min$ & $\max$ & median & $\min$ & $\max$ & SR \\
\hline$f_{1}$ & $1.33 e-06$ & $2.99 e-07$ & $4.98 e-06$ & 35998 & 34063 & 37956 & 50 \\
\hline$f_{2}$ & $5.14 \mathrm{e}-01$ & $1.71 \mathrm{e}-01$ & $1.44 \mathrm{e} 00$ & - & - & - & - \\
\hline$f_{3}$ & $1.46 e-06$ & $4.82 \mathrm{e}-07$ & $7.44 \mathrm{e}-06$ & 36079 & 33177 & 37961 & 50 \\
\hline$f_{4}$ & $4.09 e+00$ & $1.04 \mathrm{e} 00$ & $1.05 e+01$ & 190310 & 147544 & 217855 & 50 \\
\hline$f_{5}$ & $0.00 \mathrm{e} 00$ & $0.00 \mathrm{e} 00$ & $2.16 \mathrm{e}-14$ & 181779 & 137821 & 225172 & 50 \\
\hline$f_{6}$ & $6.64 e-06$ & $3.10 \mathrm{e}-07$ & $6.90 e-05$ & 86058 & 45530 & 97936 & 28 \\
\hline$f_{7}$ & $6.26 e-11$ & $3.27 e-12$ & $1.69 \mathrm{e}-08$ & 409351 & 393553 & 423387 & 50 \\
\hline$f_{8}$ & $7.55 e-15$ & $4.00 \mathrm{e}-15$ & $7.55 e-15$ & 358407 & 344448 & 374581 & 50 \\
\hline$f_{9}$ & 7.70e-01 & $3.26 \mathrm{e}-01$ & $6.16 \mathrm{e} 01$ & - & - & - & - \\
\hline$f_{10}$ & $0.00 \mathrm{e} 00$ & $0.00 \mathrm{e} 00$ & $1.04 \mathrm{e}-13$ & 152818 & 122165 & 207094 & 50 \\
\hline
\end{tabular}




\section{Table 18 (on next page)}

Comparing SS-PSO and CLPSO with Mann-Whitney tests.

+ if SS-PSO ranks first in the Mann-Whitney test and the result is significant; - if CLPSO ranks first and the results is significant; $\approx$ if the differences are not significant. 


\begin{tabular}{|c|c|c|c|c|c|c|c|c|c|c|}
\hline & $f_{1}$ & $f_{2}$ & $f_{3}$ & $f_{4}$ & $f_{5}$ & $f_{6}$ & $f_{7}$ & $f_{8}$ & $f_{9}$ & $f_{10}$ \\
\hline fitness & $\approx$ & $\approx$ & $\approx$ & $\approx$ & $\approx$ & $\approx$ & - & $\approx$ & $\approx$ & $\approx$ \\
\hline eval. & $\approx$ & $\cdot$ & $\approx$ & - & + & $\approx$ & + & - &. & + \\
\hline
\end{tabular}

1 


\section{Table $\mathbf{1 9}$ (on next page)}

DSWPSO with von Neumann neighborhood and two random neighbors 


\begin{tabular}{|c|c|c|c|c|c|c|c|}
\hline & \multicolumn{3}{|c|}{ fitness } & \multicolumn{3}{c|}{ evaluations } \\
\hline & median & $\min$ & $\max$ & $\operatorname{median}$ & $\min$ & $\max$ & SR \\
\hline $\boldsymbol{f}_{\boldsymbol{1}}$ & $8.72 \mathrm{e}-12$ & $1.07 \mathrm{e}-12$ & $5.33 \mathrm{e}-11$ & 20188 & 18767 & 22589 & 50 \\
\hline $\boldsymbol{f}_{\boldsymbol{2}}$ & $6.80 \mathrm{E}-36$ & $5.61 \mathrm{E}-39$ & $1.00 \mathrm{E}+04$ & 151704 & 121765 & 218393 & 49 \\
\hline$f_{\boldsymbol{3}}$ & $3.24 \mathrm{e}-11$ & $1.14 \mathrm{e}-12$ & $3.21 \mathrm{e}-10$ & 22981 & 20972 & 26166 & 50 \\
\hline$f_{4}$ & $6.27 \mathrm{e}+01$ & $2.69 \mathrm{e}+01$ & $1.07 \mathrm{e}+02$ & 11417 & 5586 & 31654 & 47 \\
\hline$f_{5}$ & $0.00 \mathrm{e}+00$ & $0.00 \mathrm{e}+00$ & $4.91 \mathrm{e}-02$ & 19477.5 & 17101 & 25627 & 50 \\
\hline$f_{6}$ & $0.00 \mathrm{e}+00$ & $0.00 \mathrm{e}+00$ & $9.72 \mathrm{e}-03$ & 7448 & 2989 & 28567 & 43 \\
\hline$f_{7}$ & $2.38 \mathrm{e}-02$ & $0.00 \mathrm{e}+00$ & $2.02 \mathrm{e}+00$ & 34937 & 32977 & 40180 & 20 \\
\hline$f_{\boldsymbol{8}}$ & $7.55 \mathrm{e}-15$ & $4.00 \mathrm{e}-15$ & $1.34 \mathrm{e}+00$ & 20972 & 18767 & 24892 & 47 \\
\hline$f_{9}$ & $1.43 \mathrm{e}-05$ & $6.42 \mathrm{e}-09$ & $6.63 \mathrm{e}+03$ & 639842 & 374066 & 901110 & 41 \\
\hline$f_{10}$ & $7.40 \mathrm{e}-03$ & $0.00 \mathrm{e}+00$ & $5.17 \mathrm{e}-01$ & 21021 & 18130 & 25284 & 47 \\
\hline
\end{tabular}




\section{Table $\mathbf{2 0}$ (on next page)}

DSWPSO with Moore neighborhood and two random neighbors 


\begin{tabular}{|c|c|c|c|c|c|c|c|}
\hline & \multicolumn{3}{|c}{ fitness } & \multicolumn{3}{c|}{ evaluations } \\
\hline & median & $\min$ & $\max$ & $\operatorname{median}$ & $\min$ & $\max$ & SR \\
\hline $\boldsymbol{f}_{\boldsymbol{1}}$ & $1.13 \mathrm{e}-12$ & $8.12 \mathrm{e}-14$ & $1.92 \mathrm{e}-11$ & 19306 & 17395 & 21119 & 50 \\
\hline$f_{\boldsymbol{2}}$ & $4.86 \mathrm{e}-38$ & $2.52 \mathrm{e}-41$ & $5.00 \mathrm{e}+03$ & 141708 & 121079 & 219520 & 45 \\
\hline$f_{\boldsymbol{3}}$ & $4.72 \mathrm{e}-12$ & $7.08 \mathrm{e}-13$ & $4.46 \mathrm{e}-11$ & 22050 & 19845 & 25480 & 50 \\
\hline$f_{4}$ & $6.22 \mathrm{e}+01$ & $3.48 \mathrm{e}+01$ & $1.34 \mathrm{e}+02$ & 10731 & 6958 & 23520 & 47 \\
\hline$f_{5}$ & $7.40 \mathrm{e}-03$ & $0.00 \mathrm{e}+00$ & $2.70 \mathrm{e}-02$ & 18497.5 & 16611 & 20531 & 50 \\
\hline$f_{6}$ & $0.00 \mathrm{e}+00$ & $0.00 \mathrm{e}+00$ & $9.72 \mathrm{e}-03$ & 6811 & 3136 & 25480 & 48 \\
\hline$f_{7}$ & $1.01 \mathrm{e}-01$ & $0.00 \mathrm{e}+00$ & $3.06 \mathrm{e}+00$ & 35035 & 32683 & 39494 & 16 \\
\hline$f_{\boldsymbol{8}}$ & $7.55 \mathrm{e}-15$ & $4.00 \mathrm{e}-15$ & $1.16 \mathrm{e}+00$ & 20090 & 16954 & 24941 & 47 \\
\hline$f_{\boldsymbol{g}}$ & $3.25 \mathrm{e}-05$ & $4.29 \mathrm{e}-09$ & $7.12 \mathrm{e}+03$ & 620487 & 365981 & 916692 & 35 \\
\hline$f_{10}$ & $8.63 \mathrm{e}-03$ & $0.00 \mathrm{e}+00$ & $8.00 \mathrm{e}+00$ & 19747 & 17052 & 25235 & 43 \\
\hline
\end{tabular}




\section{Table $\mathbf{2 1}$ (on next page)}

Comparing SS-PSO and DSWPSO (von Neumann) with Mann-Whitney tests.

+ if SS-PSO ranks $\square$ rst in the Mann-Whitney test and the result is signi $\square$ cant; $\square$ if DSWPSO ranks $\square$ rst and the results is signi $\square$ cant, if the differences are not significant. 


\begin{tabular}{|c|c|c|c|c|c|c|c|c|c|c|}
\hline & $f_{1}$ & $f_{2}$ & $f_{3}$ & $f_{4}$ & $f_{5}$ & $f_{6}$ & $f_{7}$ & $f_{8}$ & $f_{9}$ & $f_{10}$ \\
\hline fitness & + & + & + & $\approx$ & $\approx$ & + & + & + & + & + \\
\hline eval. & + & + & + & + & + & + & + & + & $\approx$ & + \\
\hline
\end{tabular}

1 


\section{Table 22 (on next page)}

Comparing SS-PSO and DSWPSO (Moore) with Mann-Whitney tests.

+ if SS-PSO ranks $\square$ rst in the Mann-Whitney test and the result is signi $\square$ cant; $\square$ if DSWPSO ranks $\square$ rst and the results is signi $\square$ cant, if the differences are not significant. 


\begin{tabular}{|c|c|c|c|c|c|c|c|c|c|c|}
\hline & $f_{1}$ & $f_{2}$ & $f_{3}$ & $f_{4}$ & $f_{5}$ & $f_{6}$ & $f_{7}$ & $f_{8}$ & $f_{9}$ & $f_{10}$ \\
\hline fitness & + & + & + & $\approx$ & $\approx$ & $\approx$ & + & + & + & + \\
\hline eval. & + & + & + & + & + & $\approx$ & + & + & $\approx$ & + \\
\hline
\end{tabular}

1 